\title{
Fixed-target Drell Yan -- Present \& Future
}

\author{
Wolfgang Lorenzon \\ UNIVERSITY OF MICHIGAN
}

Hadron-China 2019 Workshop, Tianjin, China

(25-August-2019)
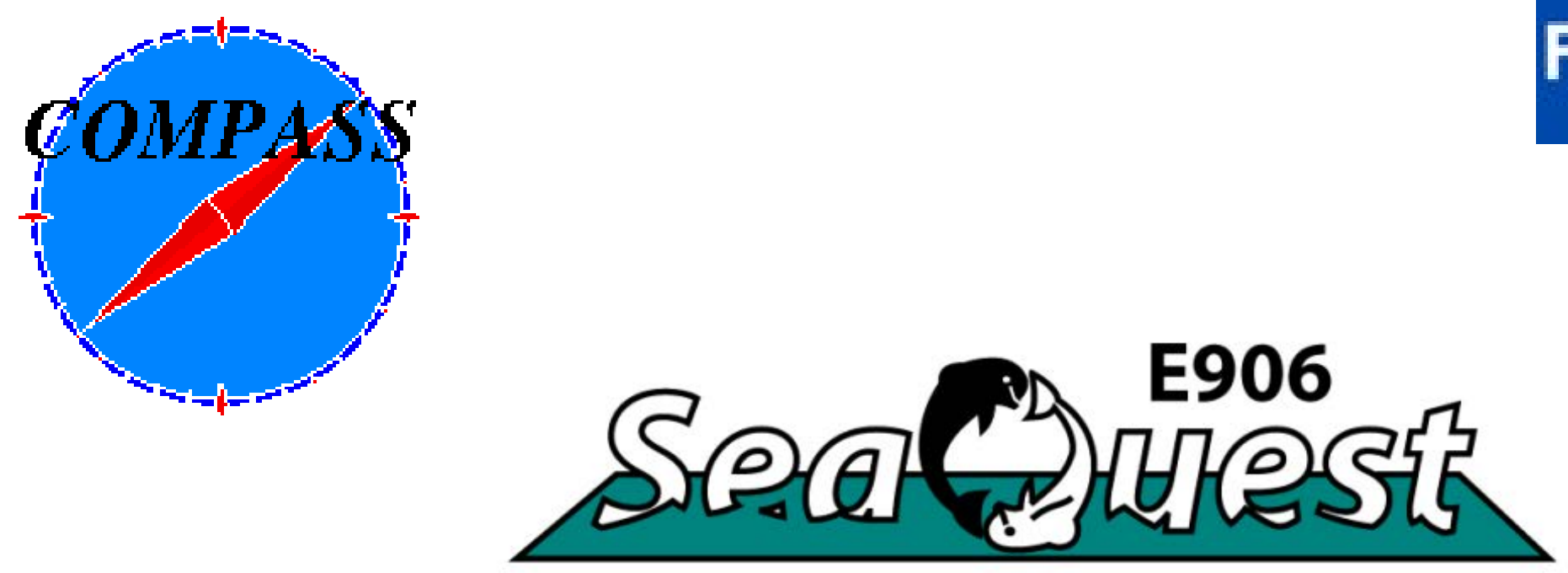

This manuscript has been authored by Fermi Research Alliance, LLC under Contract No. DE-AC02-07CH11359 with the U.S. Department of Energy, Office of Science, Office of 


\section{Complementarity between SIDIS and Drell Yan}

- SIDIS and Drell-Yan have similar physics reach:

$\rightarrow$ tools to probe quark and antiquark structure of nucleon

$\rightarrow$ electromagnetic probes

SIDIS (spacelike) virtual photon

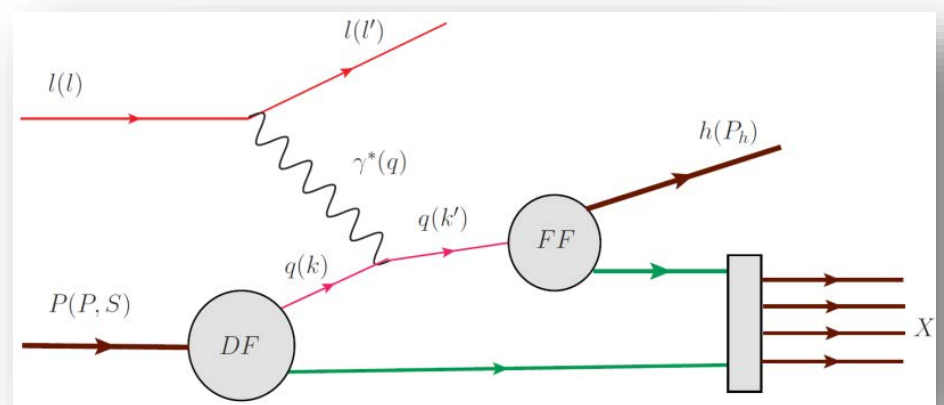

Quintessential probe of hadron structure:

$\rightarrow$ relatively simple to measure and calculate

$\rightarrow$ QCD final state effects

$\rightarrow$ fragmentation process

$\rightarrow$ no quark-antiquark selectivity
Drell-Yan (timelike) virtual photon

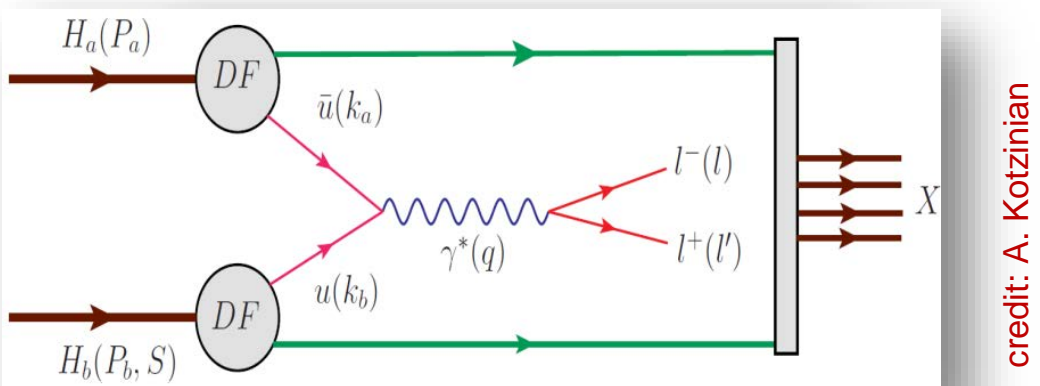

Cleanest probe to study hadron structure:

$\rightarrow$ no QCD final state effects

$\rightarrow$ no fragmentation process

$\rightarrow$ production of two TMD parton distribution functions

$\rightarrow$ ability to select sea quark distribution

$\rightarrow$ hadron beam: $\sigma(D Y) / \sigma($ nuclear $) \approx 10^{-7}$ 


\section{Factorization and Universality (SIDIS - DY)}

\author{
SIDIS \\ $\mathrm{PDF} \otimes \mathrm{FF}$
}

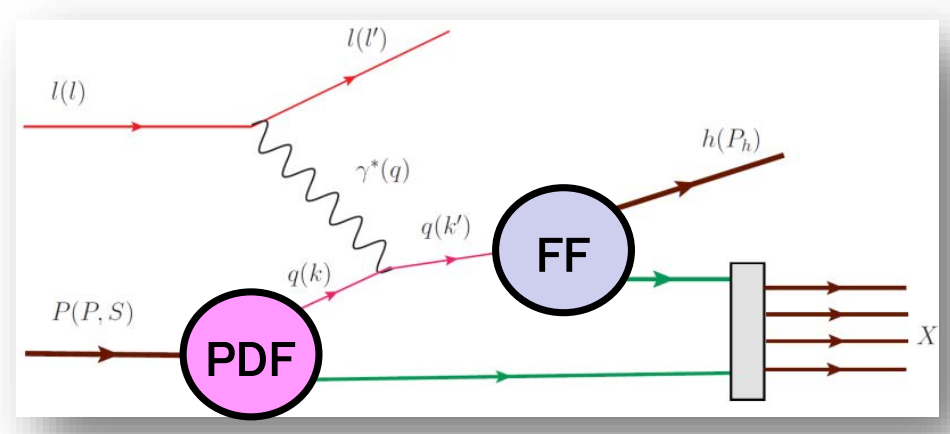

\author{
DY \\ $\mathrm{PDF} \otimes \mathrm{PDF}$
}

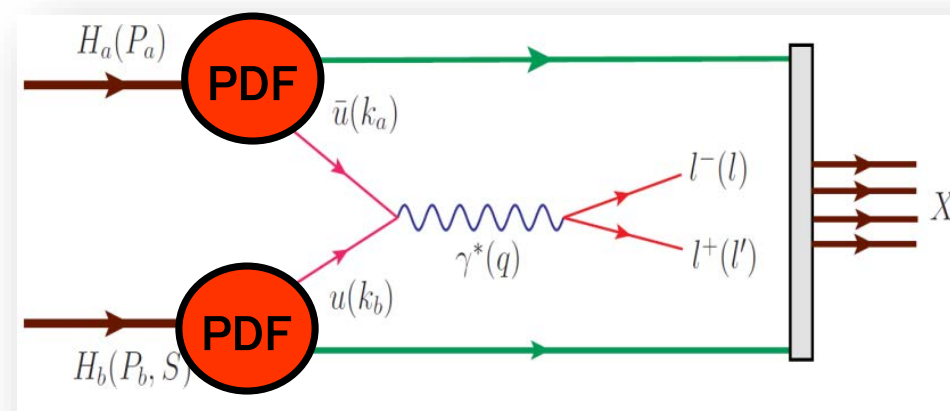

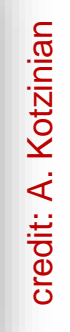

\section{Probe Universality}

are TMD PDFs in SIDIS identical to TMD PDFs in DY?

Test using unpolarized experiments, transverse SSA and DSA 


\section{LO SIDIS and single polarized DY cross sections}

\section{SIDIS}

$$
\begin{aligned}
& \frac{d \sigma_{\text {SIDIS }}^{L O}}{d x d y d z d p_{T}^{2} d \varphi_{h} d \psi}=\left[\frac{\alpha}{x y Q^{2}} \frac{y^{2}}{2(1-\varepsilon)}\left(1+\frac{\gamma^{2}}{2 x}\right)\right] \\
& \times\left(F_{U U, T}+\varepsilon F_{U U, L}\right)\left\{1+\cos 2 \phi_{h}\left(\varepsilon A_{U U}^{\cos 2 \phi_{h}}\right)\right. \\
& +\mathrm{S}_{\mathrm{T}}\left[\begin{array}{l}
\sin \left(\phi_{h}-\phi_{S}\right)\left(A_{U T}^{\sin \left(\phi_{h}-\phi_{S}\right)}\right) \\
+\sin \left(\phi_{h}+\phi_{S}\right)\left(\varepsilon A_{U T}^{\sin \left(\phi_{h}-\phi_{S}\right)}\right) \\
+\sin \left(3 \phi_{h}-\phi_{S}\right)\left(\varepsilon A_{U T}^{\sin \left(3 \phi_{h}-\phi_{S}\right)}\right)
\end{array}\right]
\end{aligned}
$$

Measure magnitude of azimuthal modulations in cross section: "Single Spin Asymmetries"

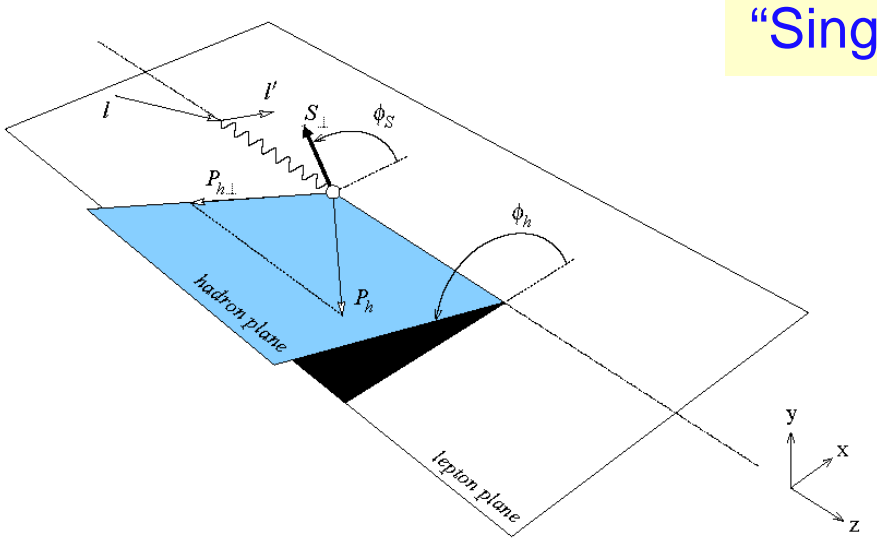

target rest frame
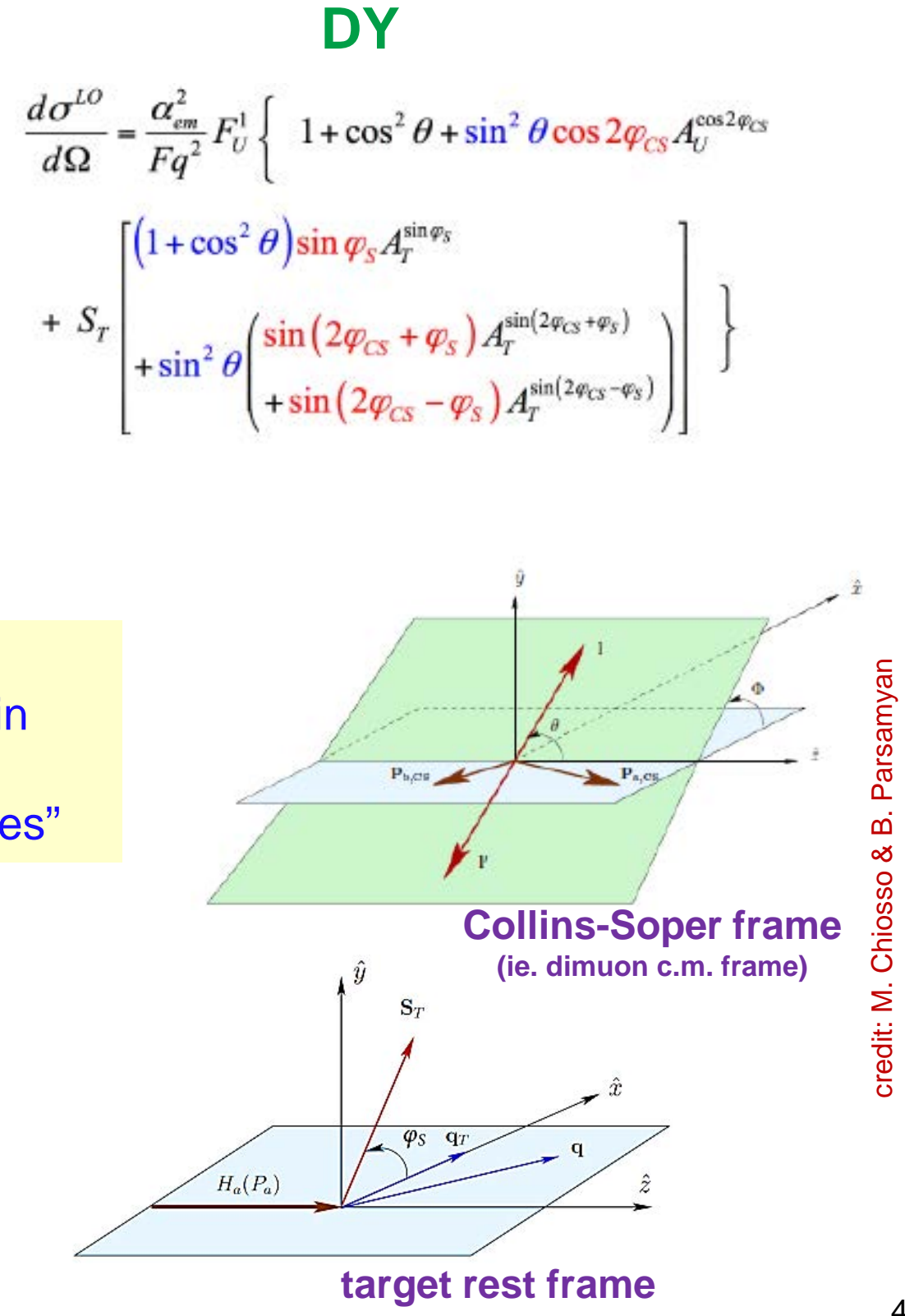


\section{LO SIDIS and single polarized DY cross sections}

\section{SIDIS}

$$
\begin{aligned}
& \frac{d \sigma_{S I D I S}^{L O}}{d x d y d z d p_{T}^{2} d \varphi_{h} d \psi}=\left[\frac{\alpha}{x y Q^{2}} \frac{y^{2}}{2(1-\varepsilon)}\left(1+\frac{\gamma^{2}}{2 x}\right)\right] \\
& \times\left(F_{U U, T}+\varepsilon F_{U U, L}\right)\left\{\begin{array}{l}
1+\cos 2 \phi_{h}\left(\varepsilon A_{U U}^{\cos 2 \phi_{h}}\right) \\
+\mathrm{S}_{\mathrm{T}}\left[\begin{array}{l}
+\sin \left(\phi_{h}+\phi_{S}\right)\left(\varepsilon A_{U T}^{\sin \left(\phi_{h}+\phi_{S}\right)}\right) \\
+\sin \left(3 \phi_{h}-\phi_{S}\right)\left(\varepsilon A_{U T}^{\sin \left(3 \phi_{h}-\phi_{S}\right)}\right)
\end{array}\right]
\end{array}\right.
\end{aligned}
$$

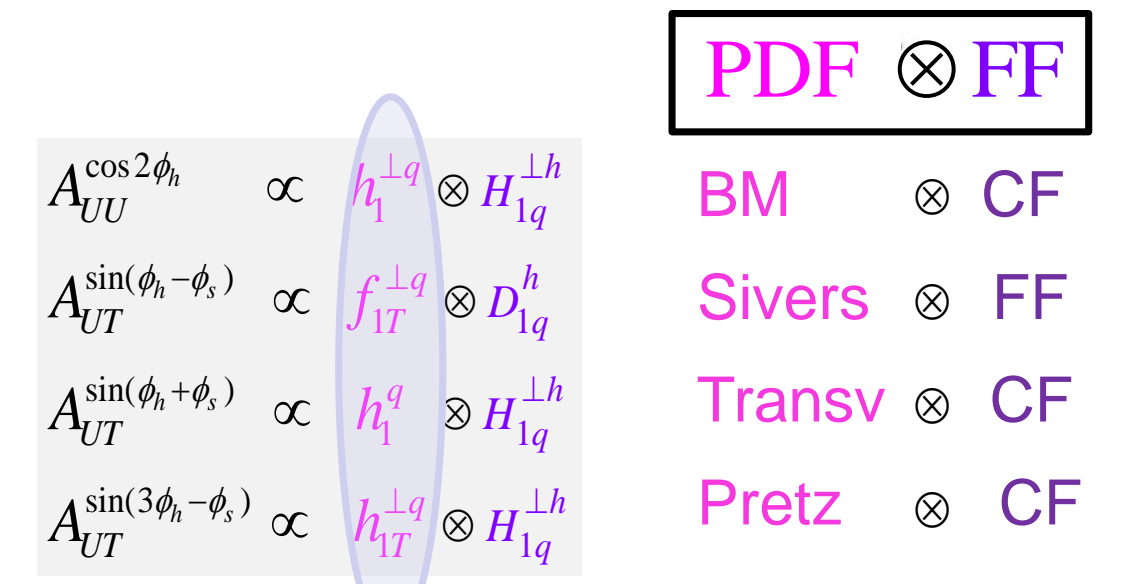

\section{DY}

$$
\begin{aligned}
& \frac{d \sigma^{L O}}{d \Omega}=\frac{\alpha_{e m}^{2}}{F q^{2}} F_{U}^{1}\left\{1+\cos ^{2} \theta+\sin ^{2} \theta \cos 2 \varphi_{C S} A_{U}^{\cos 2 \varphi_{C S}}\right. \\
& +S_{T}\left[\begin{array}{l}
\left(1+\cos ^{2} \theta\right) \sin \varphi_{S} A_{T}^{\sin \varphi_{S}} \\
\left.\left.+\sin ^{2} \theta\left(\begin{array}{l}
\sin \left(2 \varphi_{C S}+\varphi_{S}\right) A_{T}^{\sin \left(2 \varphi_{C S}+\varphi_{S}\right)} \\
+\sin \left(2 \varphi_{C S}-\varphi_{S}\right) A_{T}^{\sin \left(2 \varphi_{C S}-\varphi_{S}\right)}
\end{array}\right)\right]\right\}
\end{array}\right.
\end{aligned}
$$

beam target

$\mathrm{PDF} \otimes \mathrm{PDF}$

$\mathrm{BM} \otimes \mathrm{BM} \quad A_{T}^{\cos 2 \varphi_{c s}} \propto h_{1}^{\perp q} \otimes h_{1}^{\perp q}$

$\mathrm{f}_{1} \otimes$ Sivers $\quad A_{T}^{\sin \varphi_{s}} \propto f_{1}^{q} \otimes f_{1 T}^{\perp q}$

$\mathrm{BM} \otimes$ Transv $\quad A_{T}^{\sin \left(2 \varphi_{c s}-\varphi_{s}\right)} \propto h_{1}^{\perp q}()_{1 T}^{\perp q}$

$\mathrm{BM} \otimes$ Pretz $\quad A_{T}^{\sin \left(2 \varphi_{c s}+\varphi_{s}\right)} \propto h_{1}^{\perp q} \otimes h_{1}^{q}$

within QCD TMD framework:

$$
\begin{aligned}
& \left.h_{1}^{\perp q}\right|_{\text {SIDIS }}=-\left.h_{1}^{\perp q}\right|_{D Y} \\
& \left.f_{1 T}^{\perp q}\right|_{\text {SIDIS }}=-\left.f_{1 T}^{\perp q}\right|_{D Y}
\end{aligned}
$$$$
\begin{aligned}
& \left.h_{1}^{q}\right|_{\text {SIDIS }}=\left.h_{1}^{q}\right|_{D Y} \\
& \left.h_{1 T}^{\perp q}\right|_{\text {SIDIS }}=\left.h_{1 T}^{\perp q}\right|_{D Y}
\end{aligned}
$$ 


\section{Drell Yan Advantage}

- Complementarity is emphasized by (LO): (Arnold,Metz,Schlegel:PRD79,034005(2009))

$\rightarrow$ in SIDIS: there is $1 \mathrm{~F}_{\mathrm{U}(\mathrm{L}), \mathrm{T}}$ per TMD

$\rightarrow$ in DY:

at least 2) $F_{(U) T}$ per TMD

$\rightarrow$ same TMDs can be measured in different $F_{(U) T}$

$\rightarrow$ allowing cross checks of TMD extraction

\& even of underlying formalism

$A_{U U}^{\cos 2 \phi_{h}} \propto h_{1}^{\perp q} \otimes H_{1 q}^{\perp h}$

$A_{U T}^{\sin \left(\phi_{h}-\phi_{s}\right)} \propto f_{1 T}^{\perp q} \otimes D_{1 q}^{h}$

$A_{U T}^{\sin \left(\phi_{h}+\phi_{s}\right)} \propto h_{1}^{q} \otimes H_{1 q}^{\perp h}$

$A_{U T}^{\sin \left(3 \phi_{h}-\phi_{s}\right)} \propto h_{1 T}^{\perp q} \otimes H_{1 q}^{\perp h}$
$\mathrm{PDF} \otimes \mathrm{FF}$

BM

Sivers $\otimes$ FF

Transv $\otimes C F$

Pretz $\otimes$ CF beam target

$\mathrm{PDF} \otimes \mathrm{PDF}$

$B M \otimes B M$

$\mathrm{f}_{1} \otimes$ Sivers

$\mathrm{BM} \otimes$ Transv

BM $\otimes$ Pretz
$A_{1}^{\cos 2 \varphi_{c s}} \propto h_{1}^{\perp q} \otimes h_{1}^{\perp q}$

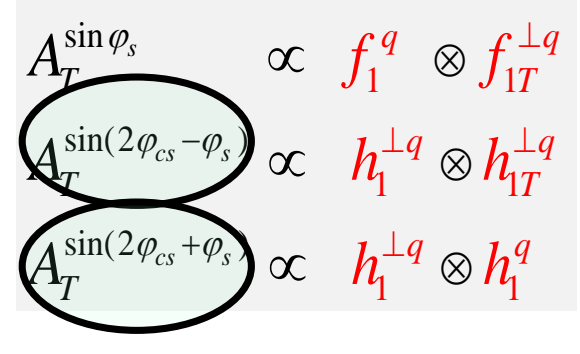

$$
A_{T}^{\sin \varphi_{s}}=\frac{F_{T}^{1}}{F_{U}^{1}}
$$




\section{Complementarity between SIDIS and Drell Yan}

- Complementarity is emphasized by (LO): (Arnold,Metz,Schlegel:PRD79,034005(2009))

$\rightarrow$ in SIDIS: there is $1 \mathrm{~F}_{\mathrm{U}(\mathrm{L}), \mathrm{T}}$ per TMD

$\rightarrow$ in DY: $\quad$ at least $2 \mathrm{~F}_{(\mathrm{U}) \mathrm{T}}$ per TMD

$\rightarrow$ same TMDs can be measured in different $F_{(U) T}$

$\rightarrow$ allowing cross checks of TMD extraction

\& even of underlying formalism TMD

- Systematic study of quark TMDs in Drell Yan

$\rightarrow$ requires double-polarization

$\rightarrow$ only then can all 8 leading twist TMD be measured

- Double-Spin Drell Yan

$\rightarrow$ Measure DY with both Beam and Target polarized $\longrightarrow$ broad spin physics program possible

$\longrightarrow$ truly complementary to spin physics programs at Jlab and RHIC and EIC 


\section{(Un)Polarized Drell Yan Experiments}

\begin{tabular}{|c|c|c|c|c|c|c|c|}
\hline Experiment & Particles & $\begin{array}{l}\text { Energy } \\
(\mathrm{GeV})\end{array}$ & $x_{b}$ or $x_{t}$ & $\begin{array}{l}\text { Luminosity } \\
\qquad\left(\mathrm{cm}^{-2} \mathrm{~s}^{-1}\right)\end{array}$ & $P_{b}$ or $P_{t}(f)$ & $\mathrm{rFOM}^{\#}$ & Timeline \\
\hline $\begin{array}{l}\text { COMPASS } \\
\text { (CERN) }\end{array}$ & $\pi^{-}+p^{\uparrow}$ & $\begin{array}{l}160 \mathrm{GeV} \\
\sqrt{\mathrm{s}}=17\end{array}$ & $x_{t}=0.1-0.3$ & $2 \times 10^{33}$ & $\begin{aligned} P_{t} & =90 \% \\
f & =0.22\end{aligned}$ & $1.1 \times 10^{-3}$ & $\begin{array}{c}2015-2016, \\
2018\end{array}$ \\
\hline $\begin{array}{l}\text { J-PARC } \\
\text { (high-p beam line) }\end{array}$ & $\pi^{-}+p$ & $\begin{array}{l}10-20 \mathrm{GeV} \\
\sqrt{s}_{s}=4.4-6.2\end{array}$ & $\begin{array}{l}x_{b}=0.2-0.97 \\
x_{t}=0.06-0.6\end{array}$ & $2 \times 10^{31}$ & --- & --- & $\begin{array}{c}>2020 ? \\
\text { under discussion }\end{array}$ \\
\hline $\begin{array}{l}\text { fsPHENIX } \\
\text { (RHIC) }\end{array}$ & $\mathbf{p}^{\uparrow}+\mathbf{p}^{\uparrow}$ & $\begin{array}{l}\sqrt{s}=200 \\
\sqrt{s}=510\end{array}$ & $\begin{array}{c}x_{b}=0.1-0.5 \\
x_{b}=0.05-0.6\end{array}$ & $\begin{array}{l}8 \times 10^{31} \\
6 \times 10^{32}\end{array}$ & $\begin{array}{l}P_{b}=60 \% \\
P_{b}=50 \%\end{array}$ & $\begin{array}{l}4.0 \times 10^{-4} \\
2.1 \times 10^{-3}\end{array}$ & $>2021 ?$ \\
\hline $\begin{array}{l}\text { SeaQuest } \\
\text { (FNAL: E-906) }\end{array}$ & $p+p$ & $\begin{array}{l}120 \mathrm{GeV} \\
\sqrt{s}=15\end{array}$ & $\begin{array}{l}x_{b}=0.35-0.9 \\
x_{t}=0.1-0.45\end{array}$ & $3.4 \times 10^{35}$ & --- & --- & $2012-2017$ \\
\hline $\begin{array}{l}\text { Pol tgt DY } \\
\text { (FNAL: E-1039) }\end{array}$ & $\begin{array}{l}p+p^{\uparrow} \\
p+d^{\uparrow}\end{array}$ & $\begin{array}{l}120 \mathrm{GeV} \\
\sqrt{\mathrm{s}}=15\end{array}$ & $x_{t}=0.1-0.45$ & $\begin{array}{l}3.0 \times 10^{35} \\
3.5 \times 10^{35}\end{array}$ & $\begin{array}{c}P_{t}=85 \% \\
f=0.176\end{array}$ & 0.15 & 2019-2021+ \\
\hline $\begin{array}{l}\text { Pol beam DY§ } \\
\text { (FNAL: E-1027) }\end{array}$ & $p^{\uparrow}+p$ & $\begin{array}{l}120 \mathrm{GeV} \\
\sqrt{\mathrm{s}}=15\end{array}$ & $x_{b}=0.35-0.9$ & $2 \times 10^{35}$ & $P_{b}=60 \%$ & 1 & $>2021 ?$ \\
\hline
\end{tabular}




\section{COMPASS 2015 Results}

- COMPASS: $190 \mathrm{GeV} \pi$ - beam on transverse polarized $\mathrm{H}$ target $\left(\mathrm{NH}_{3}\right)$

$\rightarrow 2015$ data (4 months)

$\rightarrow$ Transverse target polarization $\sim 80 \%$

$\rightarrow$ consistent $w /$ sign change!

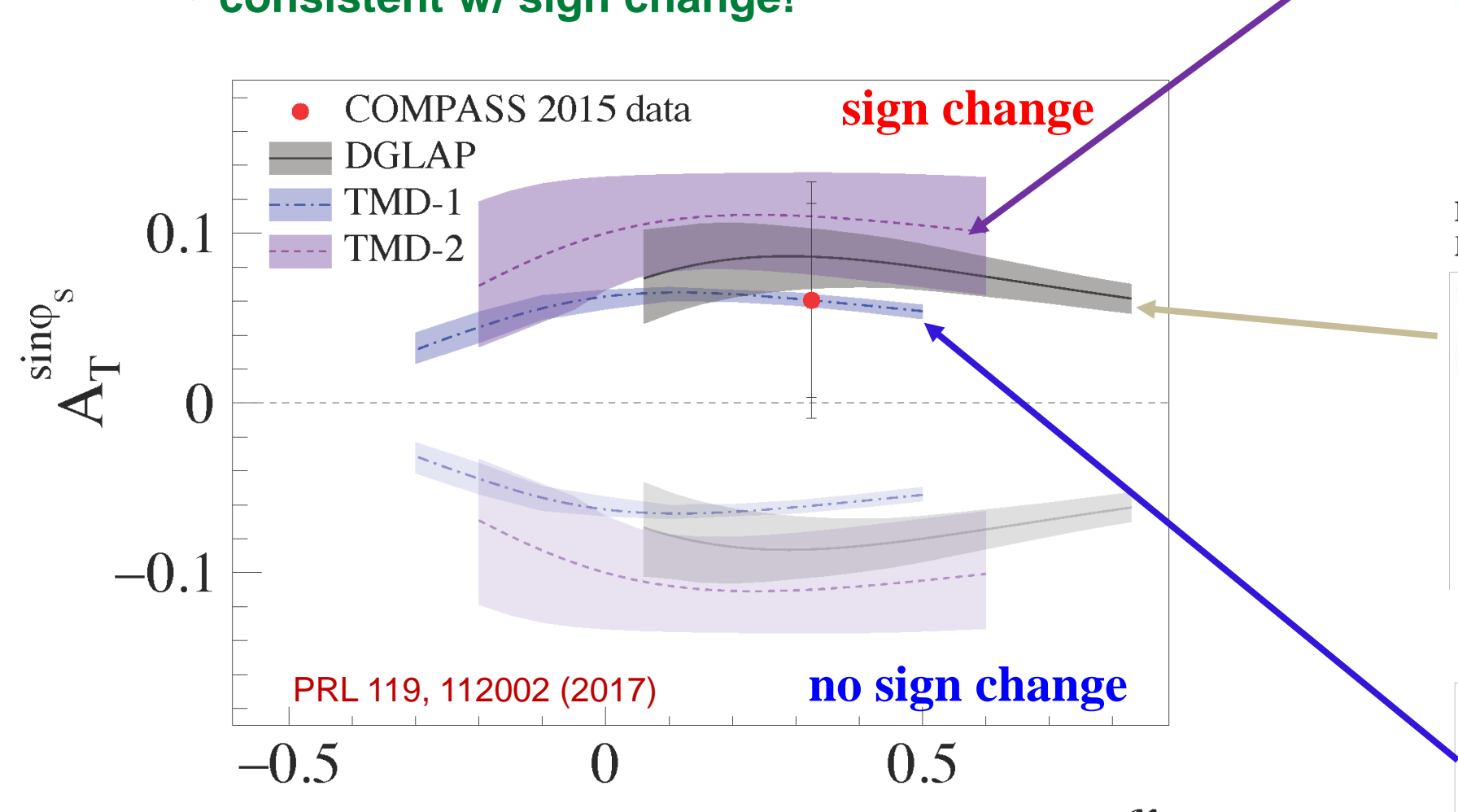

$A_{T}^{\sin \varphi_{s}}=0.060 \pm 0.057$ (stat.) \pm 0.040 (sys.)

TMD-2 (2013)

P. Sun, F. Yuan, PRD88, 114012

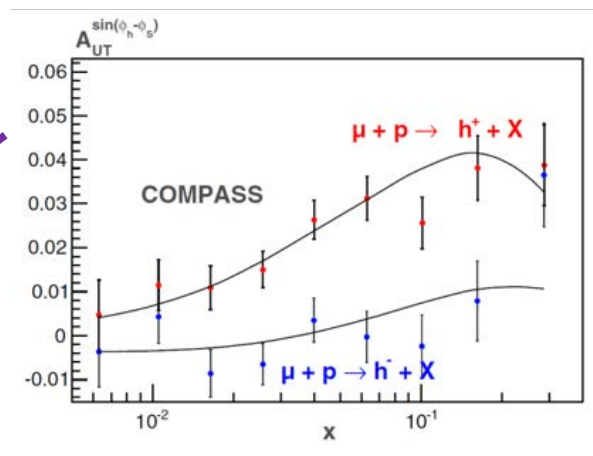

DGLAP (2016)

M. Anselmino et al. JHEP 1704, 046

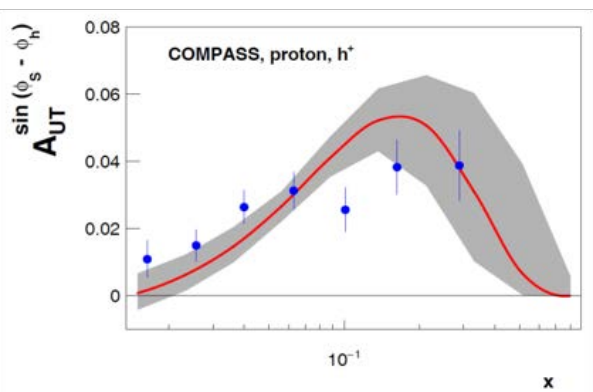

TMD-1 (2014)

M. G. Echevarria et al. PRD89,074013

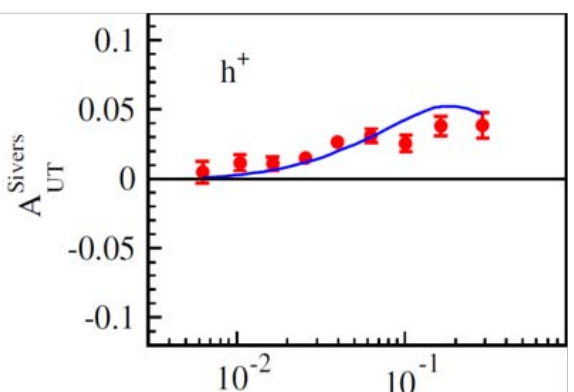




\section{Kinematic Coverage}

- Drell-Yan analysis: mass range 4.3 - 8.5 $\mathrm{GeV} / \mathrm{c}^{2}$ ("high mass range")

$\rightarrow$ only $4 \%$ background in this mass range

$\rightarrow$ DY events $\left[\mathrm{M}\left(\mu^{+} \mu^{-}\right)>4 \mathrm{GeV} / \mathrm{c}^{2}\right):-35,000$

- Phase space for Drell-Yan and SIDIS partially overlap in the $\mathrm{x}-\mathrm{Q}^{2}$ plane

$\rightarrow$ average $\mathrm{Q}^{2}$ in Drell-Yan is about $2 \mathrm{x}$ that in SIDIS

$\rightarrow$ allows to minimize the impact of uncertainties from TMD scale evolution

$\rightarrow$ overlap in kinematic regions of COMPASS Drell-Yan and SIDIS data allows for direct comparisons of TMD amplitudes

- COMPASS probes proton's valence quarks in Drell-Yan and SIDIS

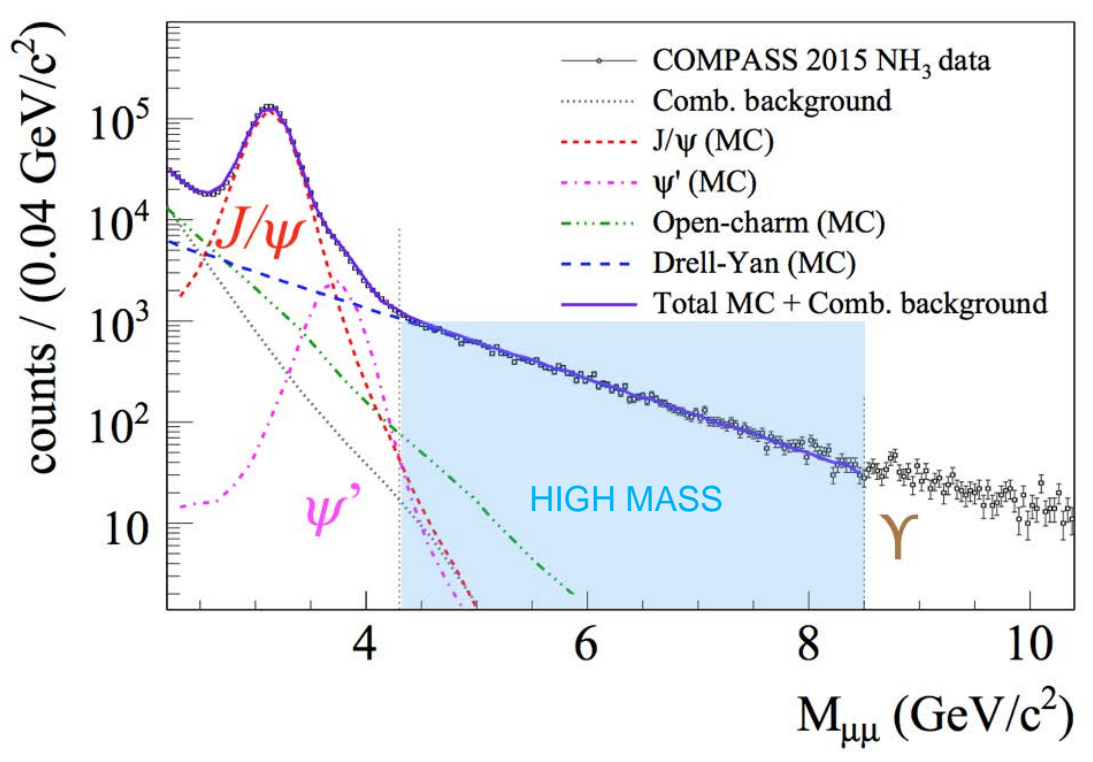

COMPASS DY / SIDIS data

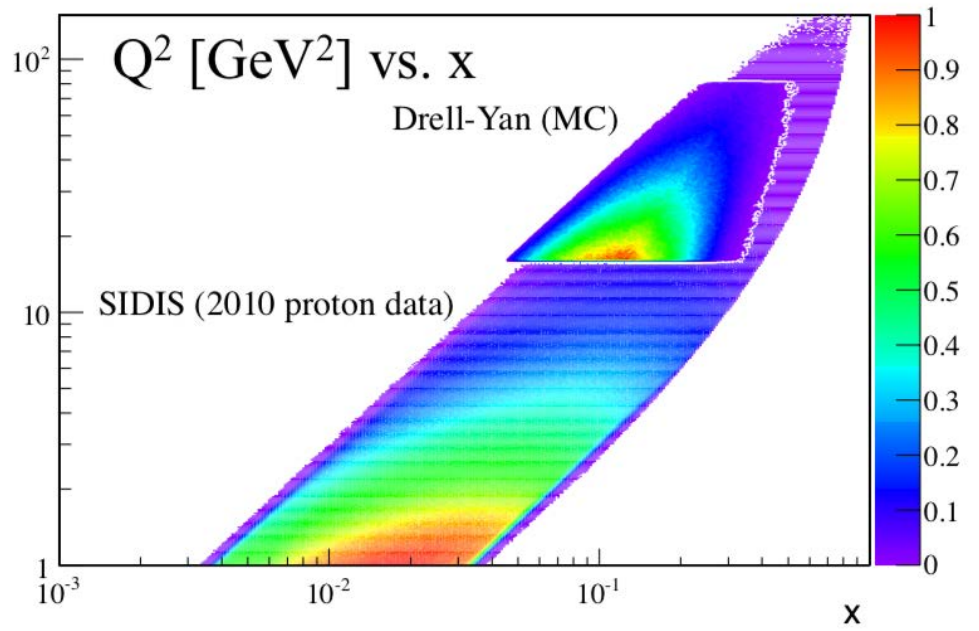




\section{Updated COMPASS Result}

- COMPASS 2015 (PRL 119 (2017) + 2018 ( $50 \%)$

$\rightarrow$ (2015 = 4 months; $2018=5$ months of data taking)

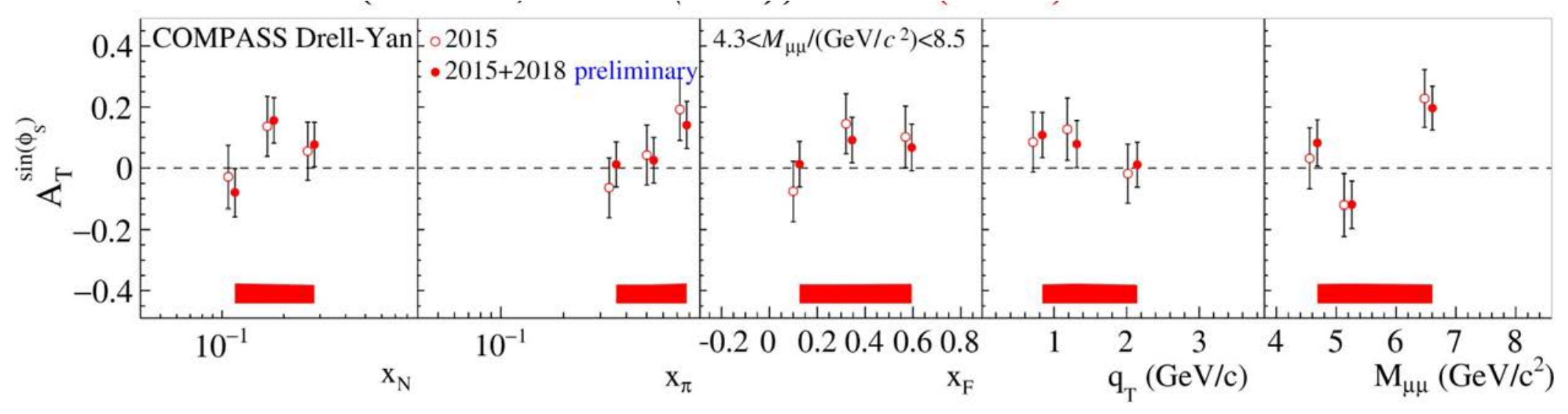

- $\mathrm{q}_{\mathrm{T}} / \mathrm{M}$ weighted asymmetries

$\rightarrow$ access to direct product of TMD PDFs

$\rightarrow$ no assumption on $\mathrm{k}_{\mathrm{T}}$ dependence of TMDs

$$
\begin{gathered}
A_{T}^{\sin \varphi_{S}} \propto f_{1, \pi}^{q} \otimes f_{1 T, p}^{\perp q} \\
A_{T}^{\sin \varphi_{S} \frac{q_{T}}{M_{N}}} \propto f_{1, \pi}^{q} \times f_{1 T, p}^{\perp q(1)}
\end{gathered}
$$

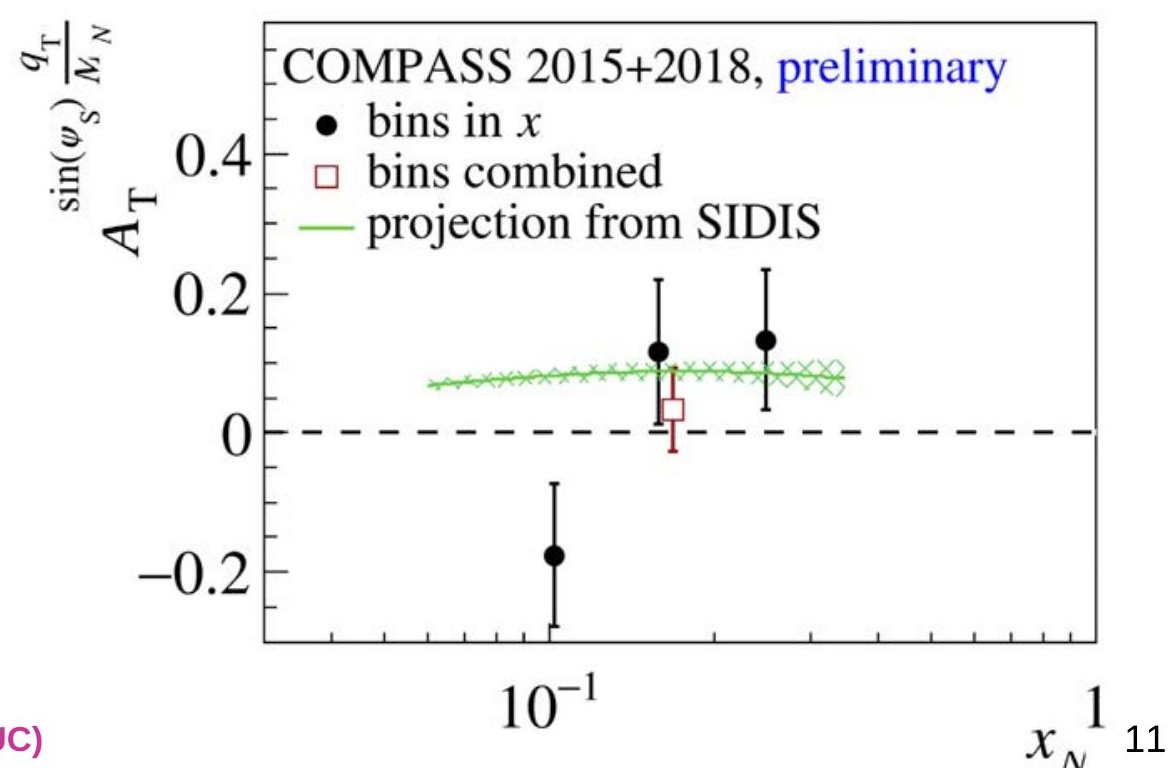




\section{COMPASS Plans (DY)}

- Sivers asymmetry:

$\rightarrow$ Sivers asymmetry measured both in polarized Drell-Yan and SIDIS processes with the same apparatus

$\rightarrow$ no more sign-change data

- Unpolarized DY:

$\rightarrow$ valence quark distributions for pion

$\rightarrow$ Boer-Mulders TMD extraction

$\rightarrow$ nuclear dependence (EMC effect, Energy loss and Cronin effect)

$\rightarrow$ detailed study of the fundamental Lam-Tung relation violation

- COMPASS++ I AMBER (DY program) (LOI $\rightarrow$ Proposal: 2021+)

$\rightarrow$ main objectives: improve significantly our knowledge of pion and kaon PDFs (2024)

$\rightarrow$ plan to run with radio separated kaon/anti-p beam for DY and spectroscopy (>2025)

$\checkmark$ nucleon spin structure with anti-p beam on transversely polarized target

$\checkmark$ flavor separation of TMD SSAs

$\checkmark$ gluon TMDs in kaon

$\checkmark$ direct measurement of the lifetime of neutral pion 


\section{Sivers Program at STAR}

- RHIC p+p (500 GeV): $W^{+/-}$TSSA

$$
\begin{aligned}
& \mathrm{A}_{\mathrm{N}}\left(\mathrm{W}^{+}\right) \sim\left(\Delta^{N} f_{u / p^{\uparrow}} \otimes f_{\bar{d} / p}+\Delta^{N} f_{\bar{d} / p^{\uparrow}} \otimes f_{u / p}\right) \\
& \mathrm{A}_{\mathrm{N}}\left(\mathrm{W}^{-}\right) \sim\left(\Delta^{N} f_{\bar{u} / p^{\uparrow}} \otimes f_{d / p}+\Delta^{N} f_{d / p^{\uparrow}} \otimes f_{\bar{u} / p}\right)
\end{aligned}
$$

- Sivers asymmetry:

$\rightarrow$ quark flavor identified

$\rightarrow$ high $\mathrm{Q}^{2}$

$\rightarrow$ statistically limited: $\mathrm{O}(10 \%)$

$\rightarrow$ data favor sign-change if TMD evolution effects small

$\rightarrow$ more data from $2017\left(400 \mathrm{pb}^{-1}\right)$ soon
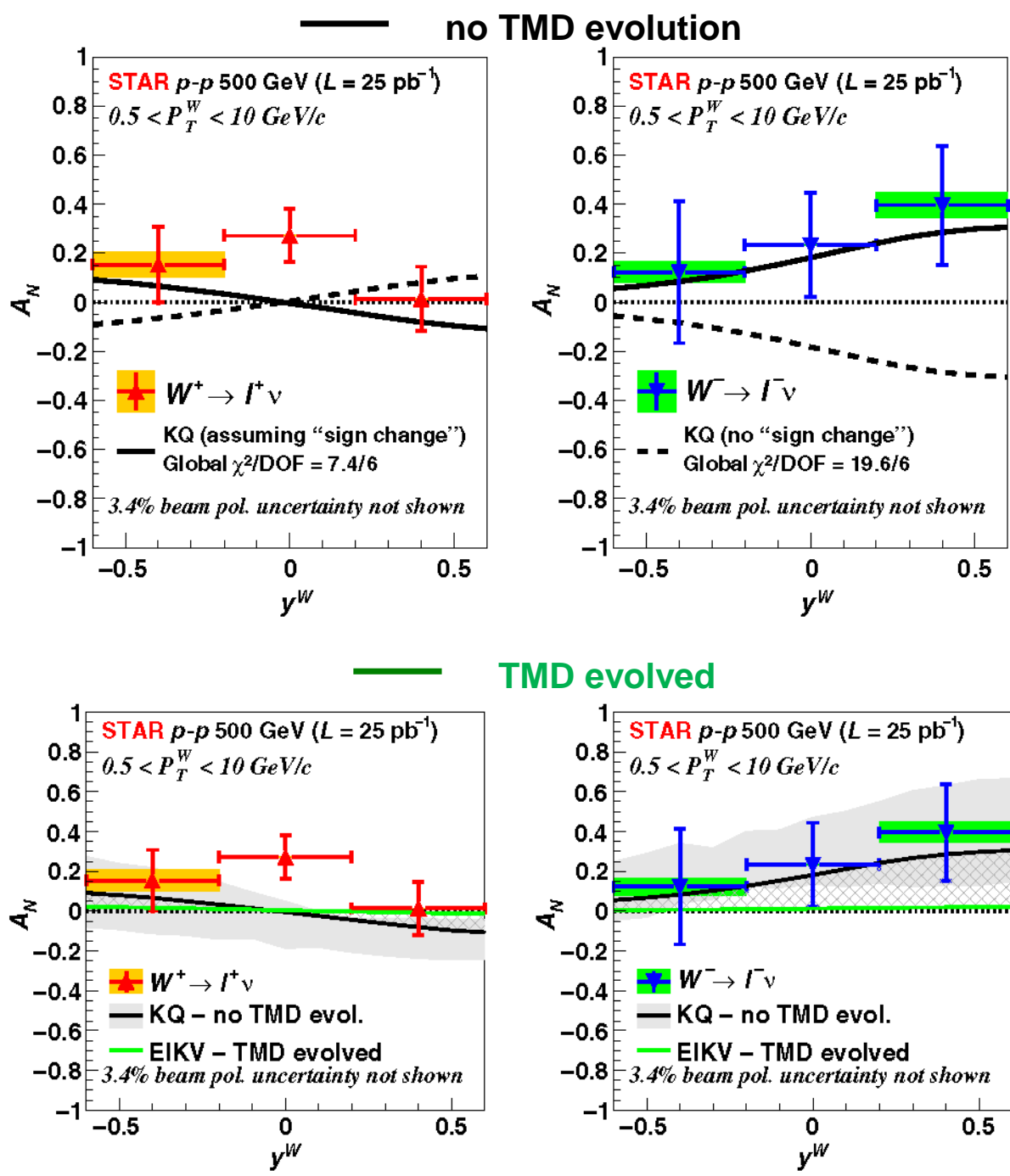

TMD evolved

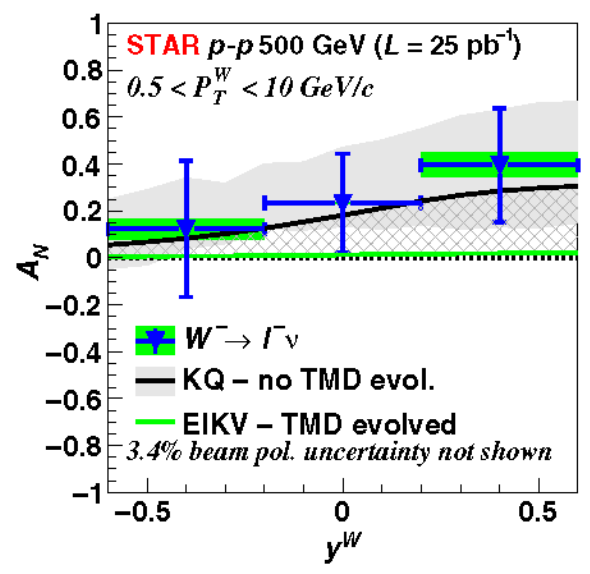

PRL 116 (2016) 132301 


\section{Recent, Current and Future DY Program at FNAL}

Unpolarized Beam and Target w/ SeaQuest detector

- E-906/SeaQuest: $120 \mathrm{GeV}$ p from Main Injector on $\mathrm{LH}_{2}, \mathrm{LD}_{2}, \mathrm{C}, \mathrm{Fe}, \mathrm{W}$ targets

$\rightarrow$ high-x Drell Yan

- Science run: March 2014 - July 2017

$\rightarrow$ dbar/ubar asymmetry, nuclear dependence, quark energy loss, Tam-Tung relation,...

Unpolarized Beam and polarized Target ( $w /$ upgraded SeaQuest detector)

- E-1039/SpinQuest: SeaQuest w/ pol $\mathrm{NH}_{3} / \mathrm{ND}_{3}$ targets: 2019-2021

$\rightarrow$ probe sea quark distributions

Polarized Beam and polarized Target

$\rightarrow$ development of high-luminosity facility for polarized Drell Yan

- E-1027: pol p beam on (un)pol tgt (2021+?)

$\rightarrow$ Sivers sign change (valence quark)

$\rightarrow$ TMD physics program complementary to future EIC program

Other opportunities

- E-1067/DarkQuest

$\rightarrow$ parasitic dark photon search (2016-2021+)

$\rightarrow$ dedicated run? (2021+?) 


\section{SeaQuest Experiment}

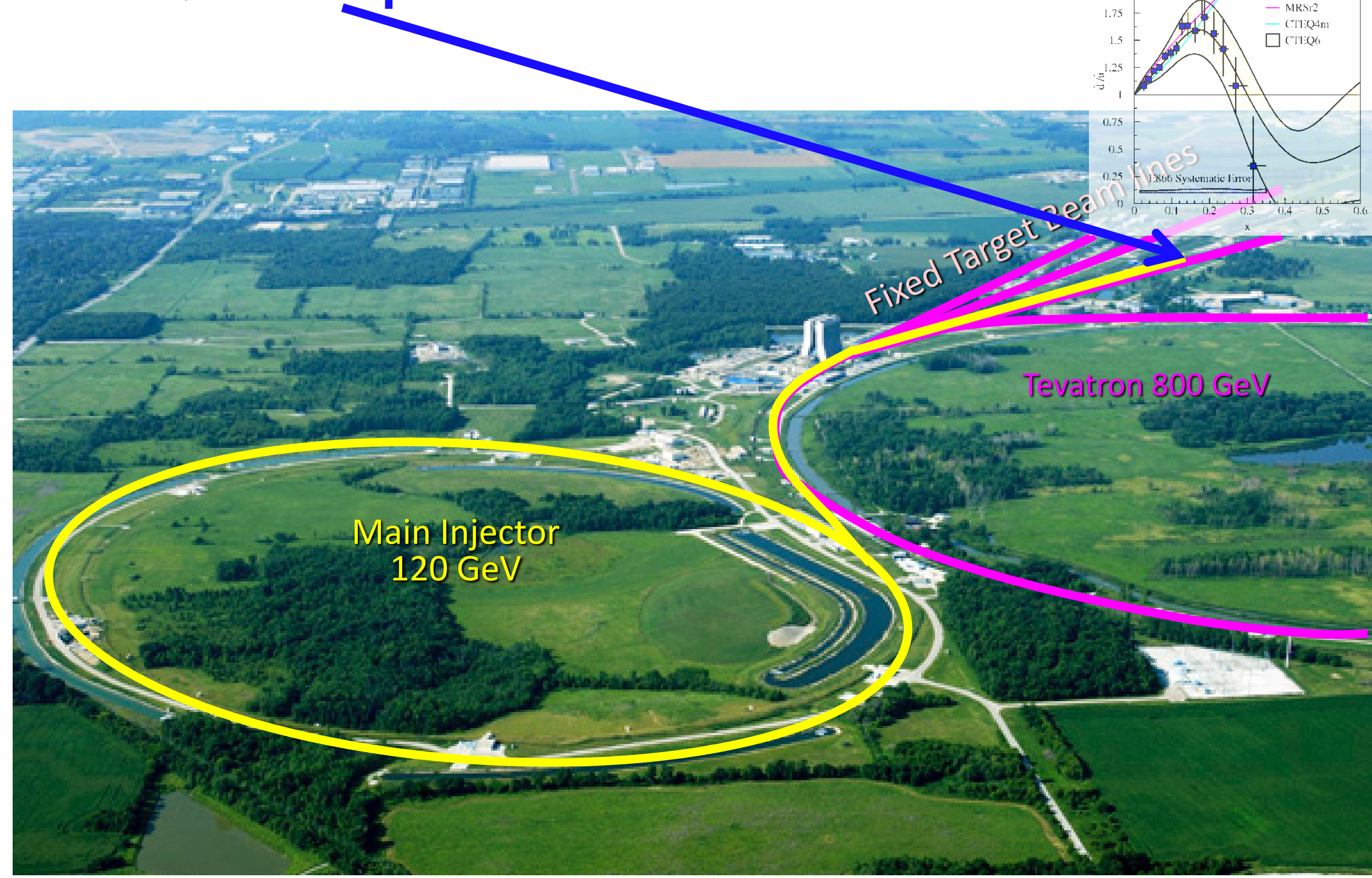

$10 \%$ of available beam to SeaQuest I $90 \%$ to neutrino program 


\section{SpinQuest Spectrometer}

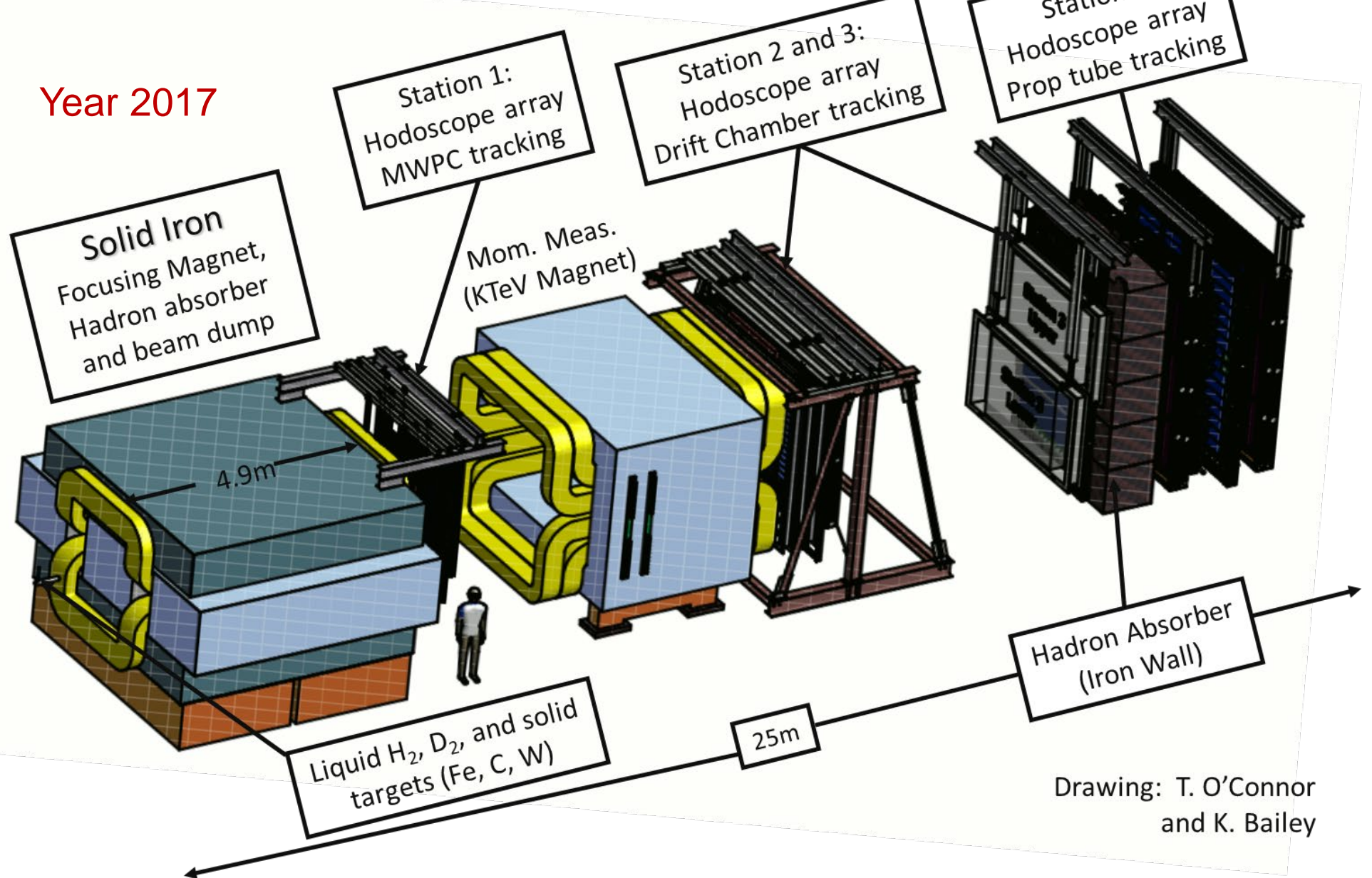

$120 \mathrm{GeV}$ protons from the Main Injector

- 4.3s beam spill every $60 \mathrm{sec}$

- 19ns RF, 10Ks p/RF bucket

- $5 \times 10^{12} \mathrm{p} / \mathrm{spill}$

- Total integrated POT for E1039 (2-year): $1.4 \times 10^{18}$ POT 


\section{Dimuon Mass from SeaQuest}

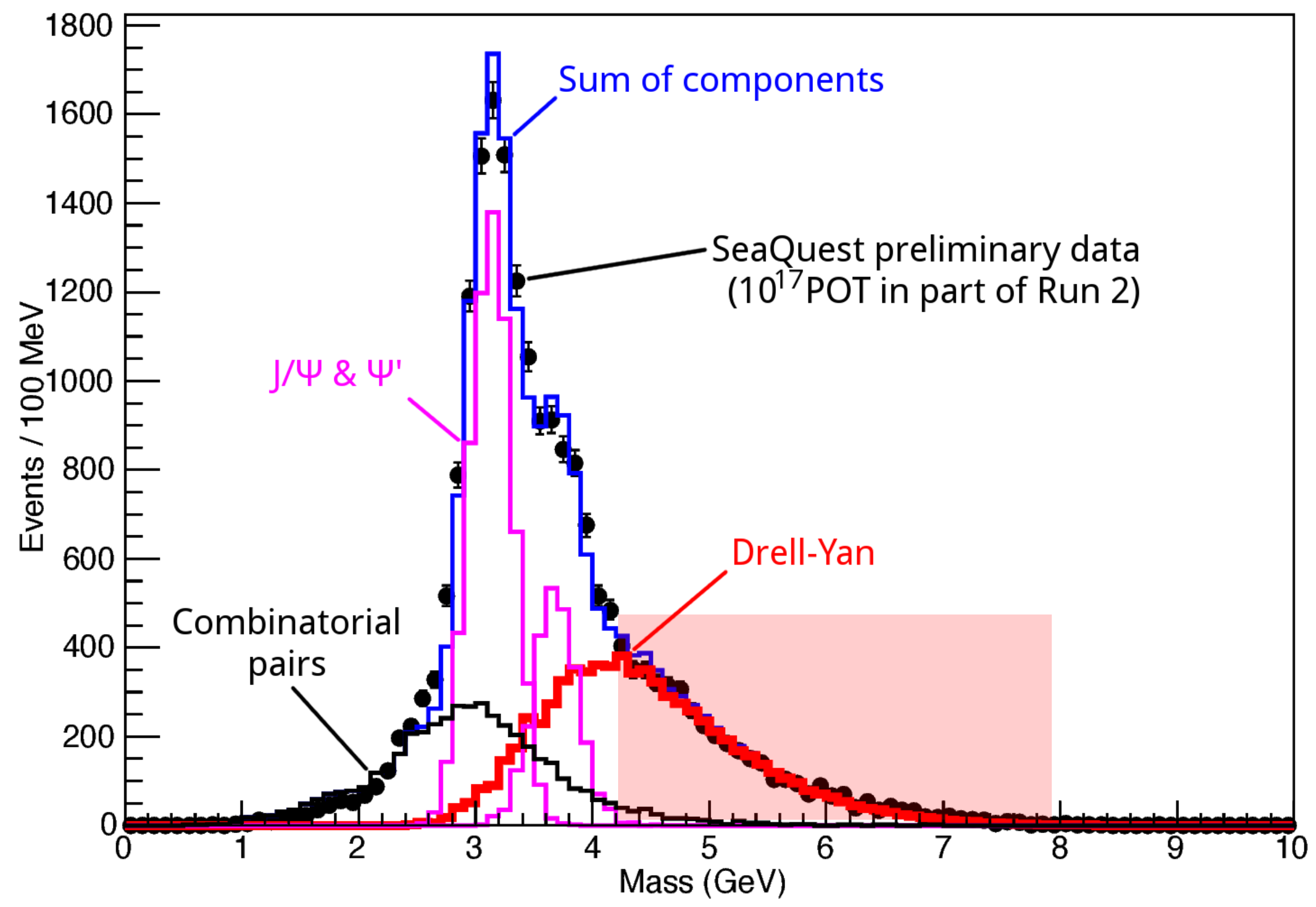




\section{Fixed Target DY at SeaQuest: A Sea Quark Laboratory}
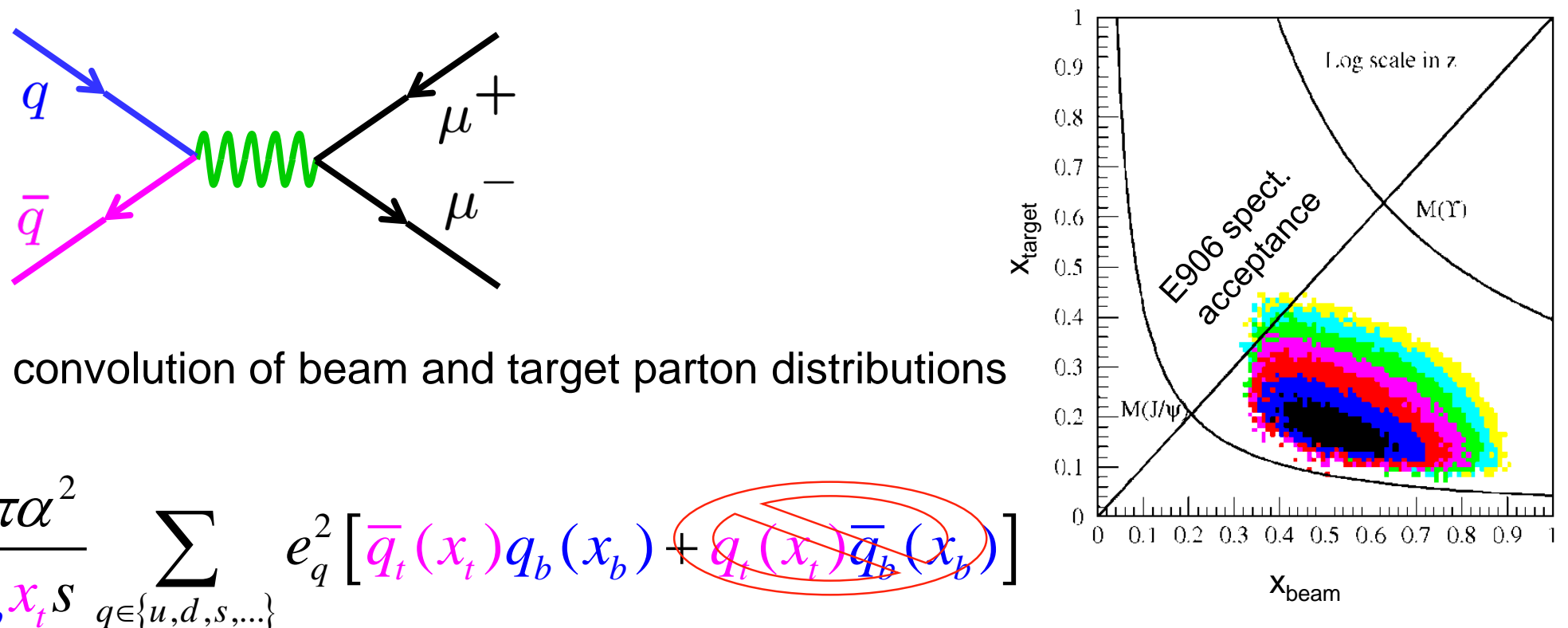

$\frac{d^{2} \sigma}{d x_{b} d x_{t}}=\frac{4 \pi \alpha^{2}}{x_{b} x_{t} s} \sum_{q \in\{u, d, s, \ldots\}} e_{q}^{2}\left[\bar{q}_{t}\left(x_{t}\right) q_{b}\left(x_{b}\right)+\widetilde{q_{t}\left(x_{t}\right) \bar{q}_{b}\left(x_{b}\right)}\right]$

beam: valence quarks at high $\mathrm{x}$

$\frac{\sigma^{\mathrm{pd}}}{2 \sigma^{\mathrm{pp}}}=\frac{1}{2}\left[1+\frac{\bar{d}(x)}{\bar{u}(x)}\right]$

target: sea quarks at low/intermediate $\mathrm{x}$

Fixed target kinematics favors sea-quarks from target - a sea quark laboratory! 


\section{Flavor Asymmetry of SeaQuarks}

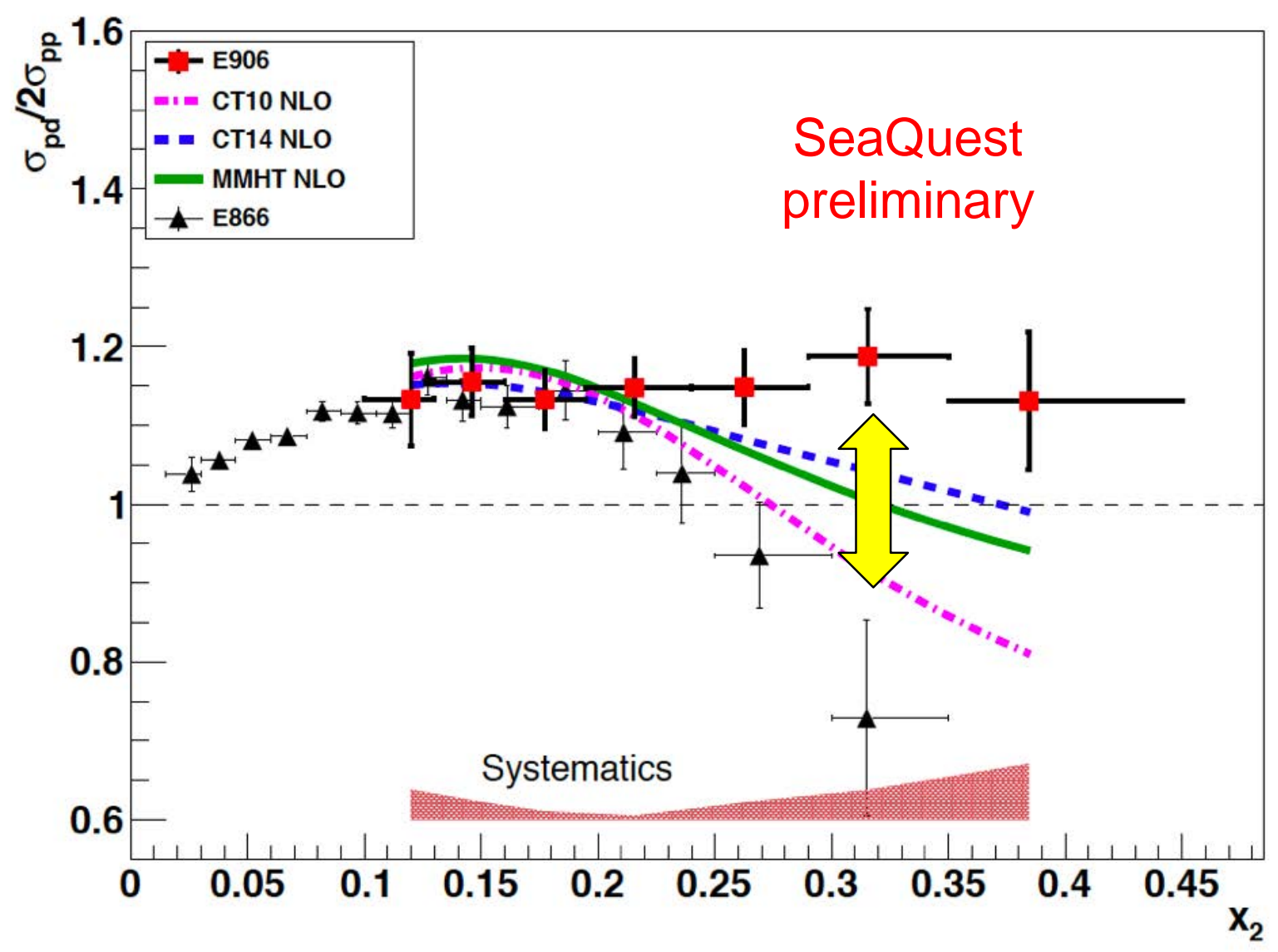

- E866 data is for $\mathrm{Q}^{2}=54 \mathrm{GeV}^{2}$ while SeaQuest data has $\mathrm{Q}^{2} \approx 29 \mathrm{GeV}^{2}$

- difference should be insignificant

- is there disagreement at high $x$ ?

- dbar / ubar coming soon! 


\section{Polarized Target Developed for DY Sivers}

- field: $5 \mathrm{~T} @ 1 \mathrm{~K}$

- targets: $\mathrm{NH}_{3}$ and $\mathrm{ND}_{3}$

- elliptical: $27 \mathrm{~mm}$ x $20 \mathrm{~mm}(\mathrm{x}, \mathrm{y}), \mathrm{l}: 80 \mathrm{~mm}(\mathrm{z})$

- 2 active cells, 1 empty

- helium consumption 200 I/day
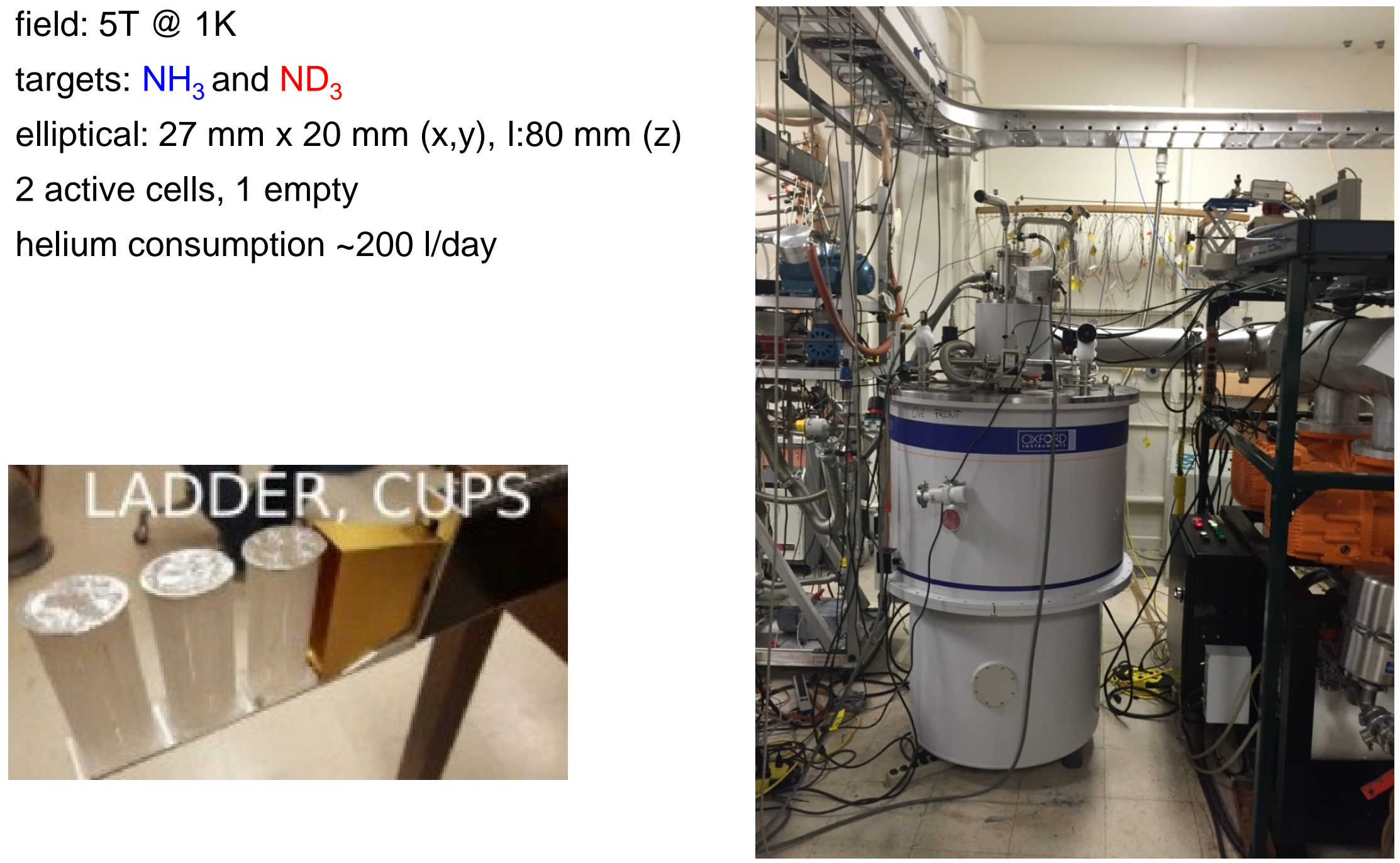


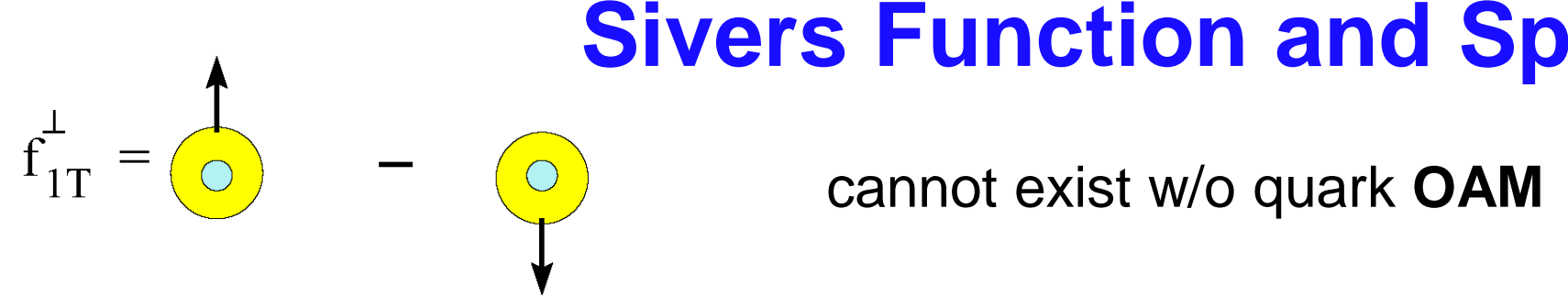

- describes transverse-momentum distribution of unpolarized quarks inside transversely polarized proton

- connection b/w Sivers function and OAM is yet model-dependent

$$
\begin{array}{l|l}
\frac{1}{2}=\frac{1}{2} \Delta \Sigma+\Delta G+L & 1 / 2 \Delta \Sigma \approx 25 \% ; \Delta G \approx 20 \% \\
\Delta \Sigma=\Delta u+\Delta d+\Delta s & L \approx \text { unmeasured }
\end{array}
$$

\section{How measure quark OAM ?}

- GPD: Generalized Parton Distribution

- TMD: Transverse Momentum Distribution

$$
A_{N}^{D Y} \propto \frac{u\left(x_{b}\right) \cdot f_{1 T}^{\perp, \bar{u}}\left(x_{t}\right)}{u\left(x_{b}\right) \cdot \bar{u}\left(x_{t}\right)}
$$

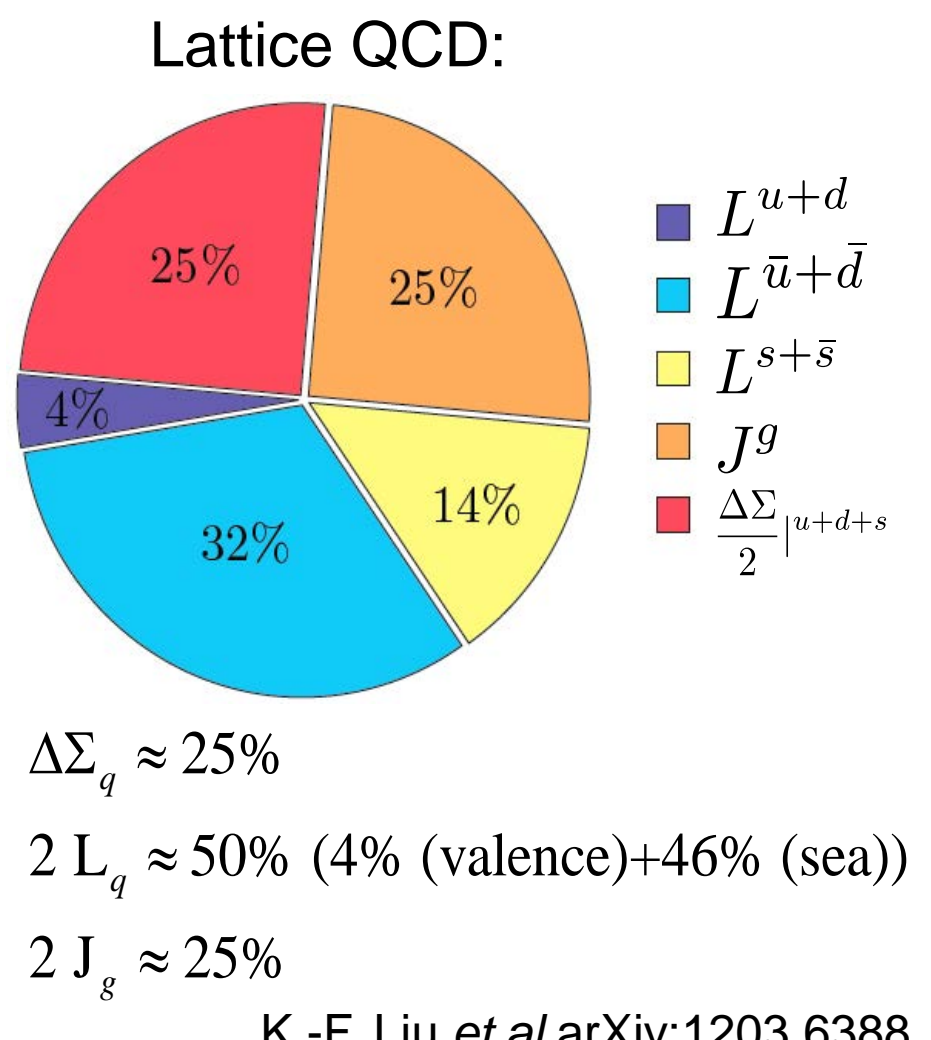




\section{Projected DY Transverse Single Spin Asymmetry}

\section{E1039 proposal}

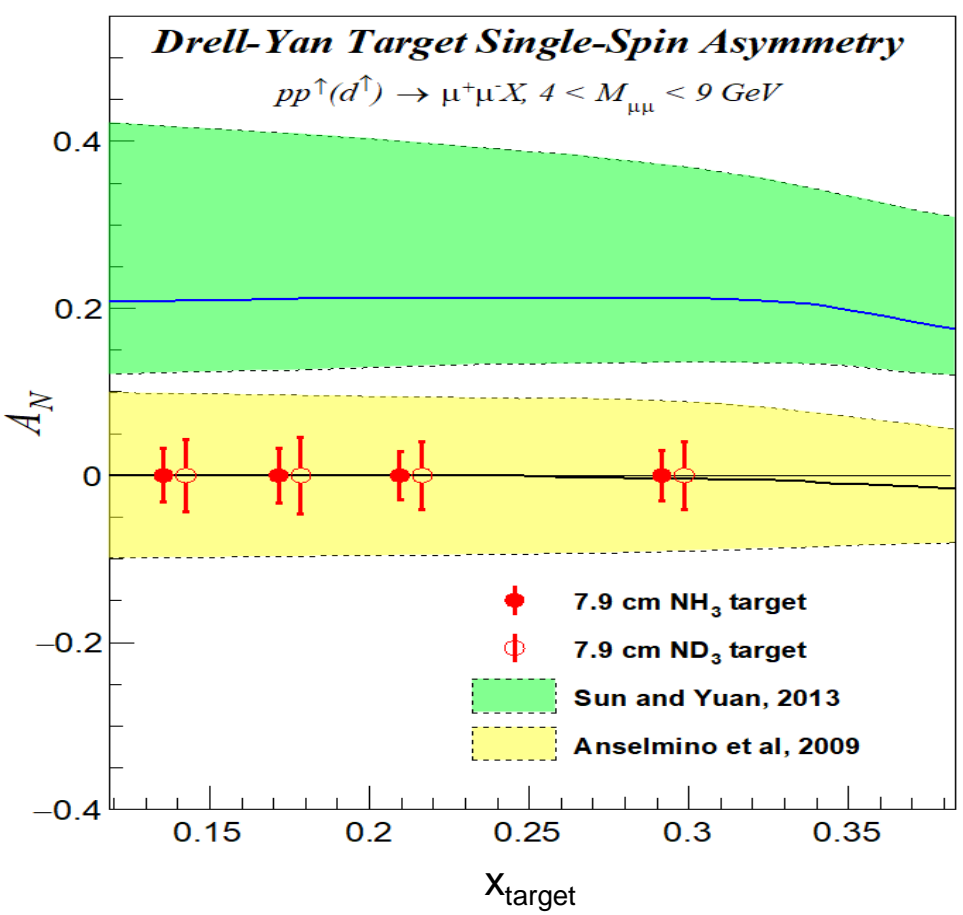

- existing SIDIS data poorly constrain sea-quark Sivers function

(Anselmino)

- significant Sivers asymmetry expected from meson-cloud model (Sun \& Yuan)
More recent calculations
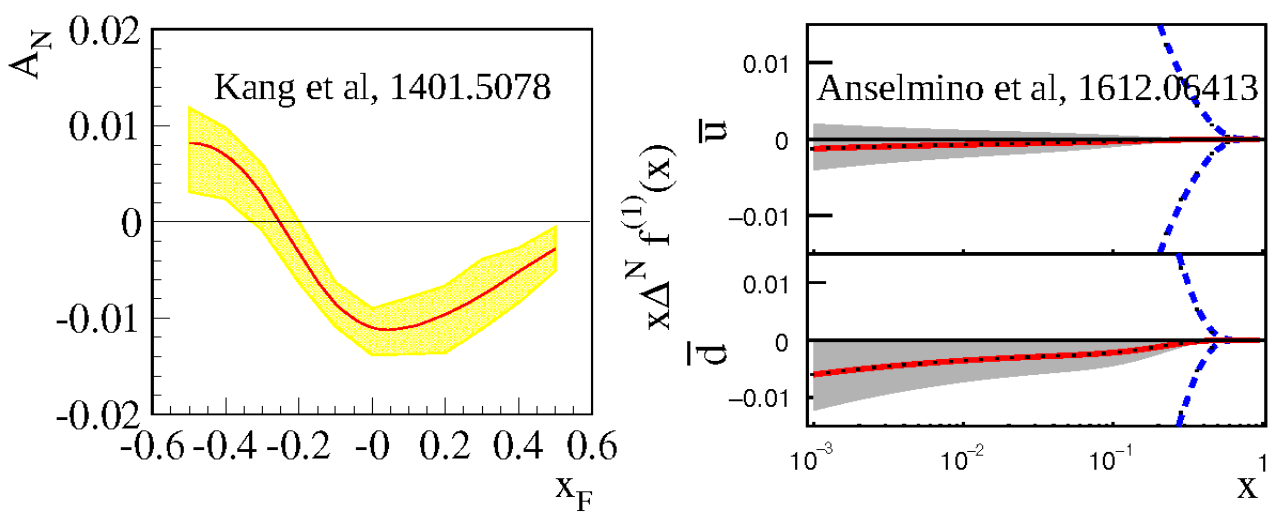

- determine sign and value of sea quark Sivers asymmetry

- measure sea quark Sivers flavor dependence (H \& D targets)

If $A_{N} \neq 0$, major discovery:

"Smoking Gun" evidence for $\mathrm{L}_{\bar{u}, \bar{d}} \neq 0$ 


\section{E1039 Status \& Plans}

- DOE approval, March 2018

- Fermilab stage-2 approval, May 2018

- E906 decommissioned, June 2018

- Polarized target to be installed by fall of 2019

- E1039 commissioning starts in late 2019

- Run for 2+ years, 2019-2021+

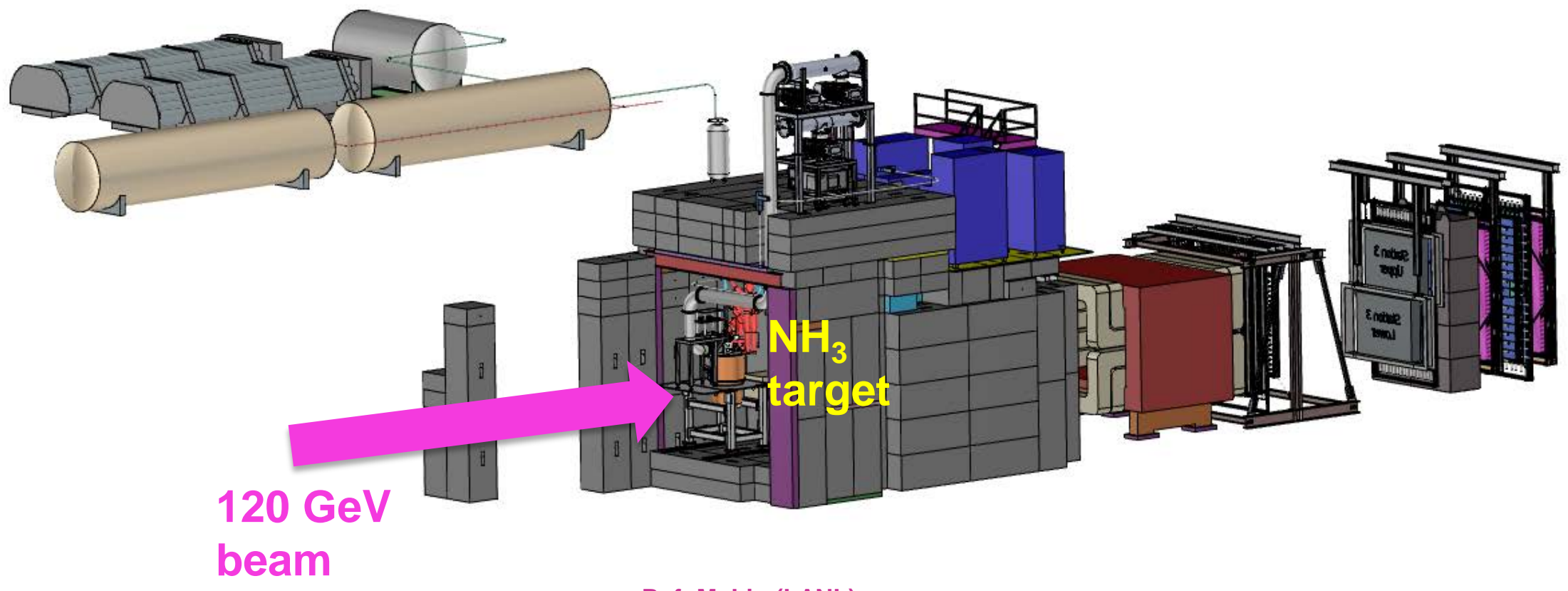




\section{E1039 Milestones - Fermilab}

- major modifications in experimental hall (special thanks to Fermilab)

$\rightarrow$ beamline: new collimator

$\rightarrow$ new radiation shielding design

$\rightarrow$ new cryo platform for polarized target infrastructure

$\rightarrow$ polarized target cave: new location $300 \mathrm{~cm}$ upstream of FMAG

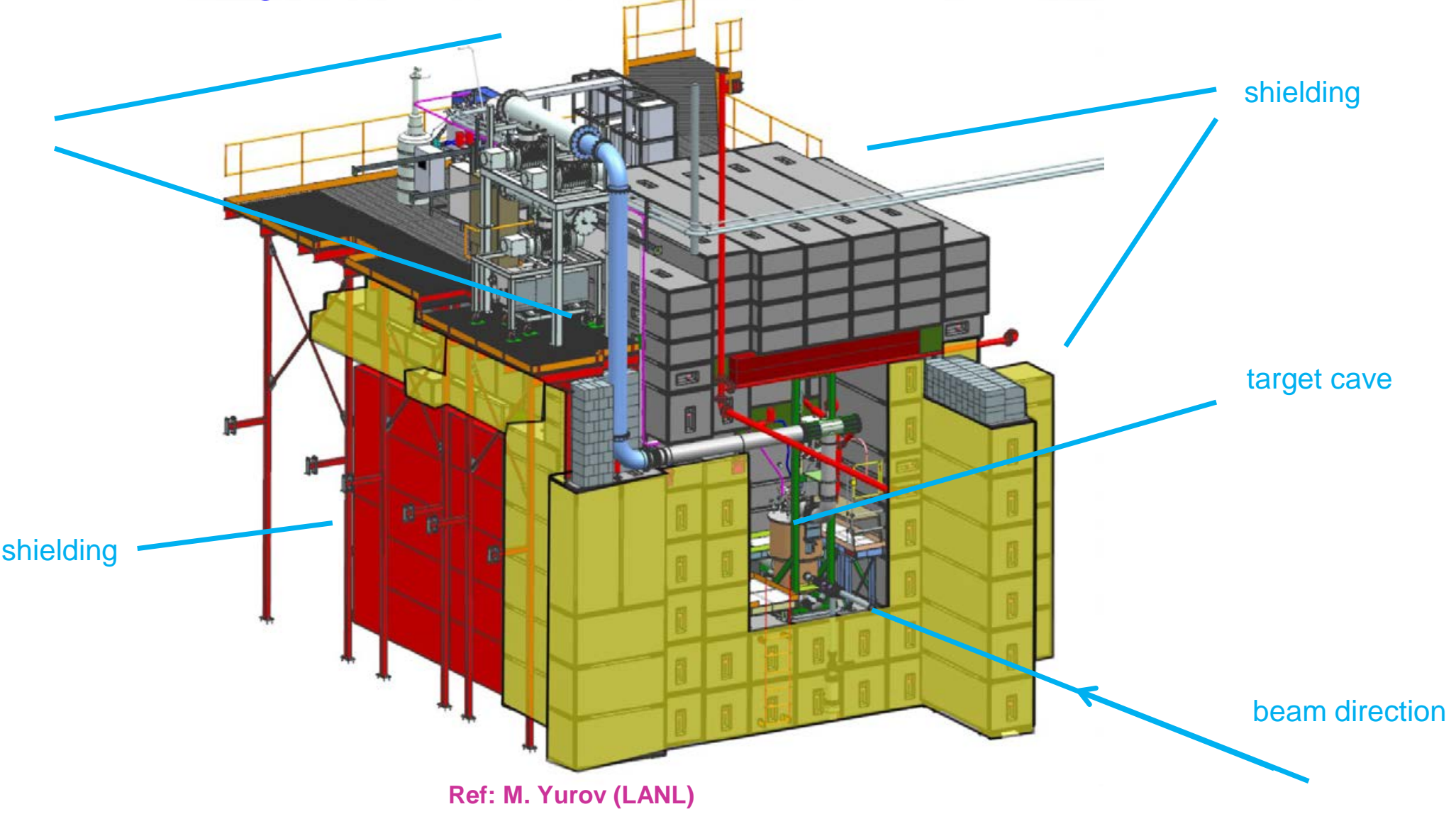




\section{E1039 Milestones - Collaboration}

- new polarized target construction: LANL and UVA effort

$\rightarrow$ rebuild magnet; change field direction

$\rightarrow$ rebuild high cooling power evaporation refrigerator

$\rightarrow$ build two new polarized target inserts

$\rightarrow$ new pump set; high cooling capacity

$\rightarrow$ new high power microwave source

$\rightarrow$ polarization of $\mathrm{NH}_{3}>90 \%$

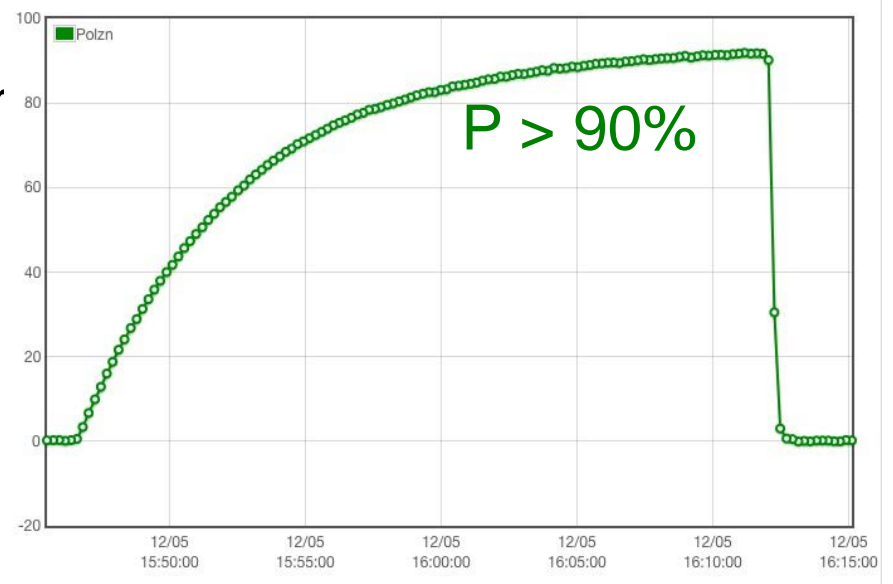

- new NMR system

$\rightarrow$ replacing Liverpool Q-meter

$\rightarrow$ cold NMR

- He liquefier for liquid helium recirculation

$\rightarrow$ constructed by "Quantum technologies"

$\rightarrow \sim 200$ L/day capacity

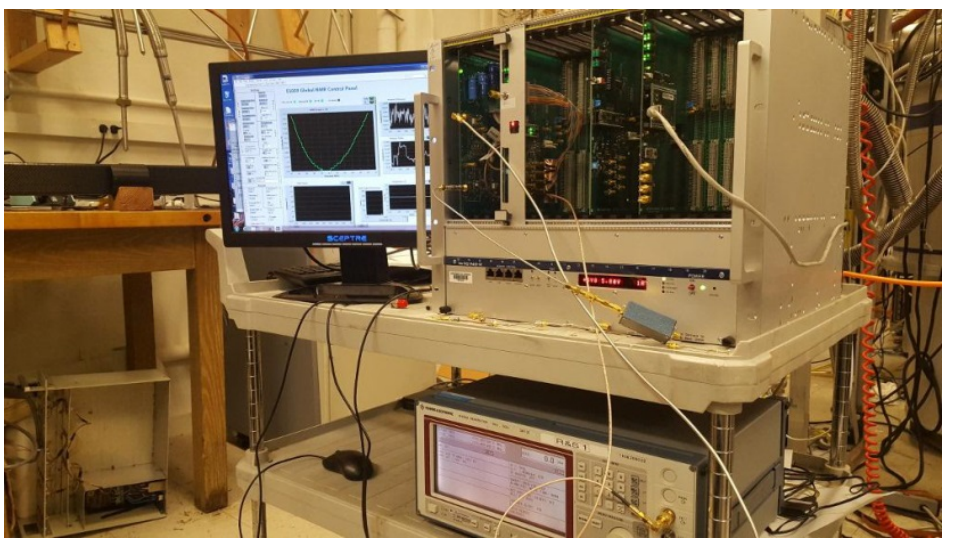

new LANL NMR setup 


\section{E1039 Collaboration}

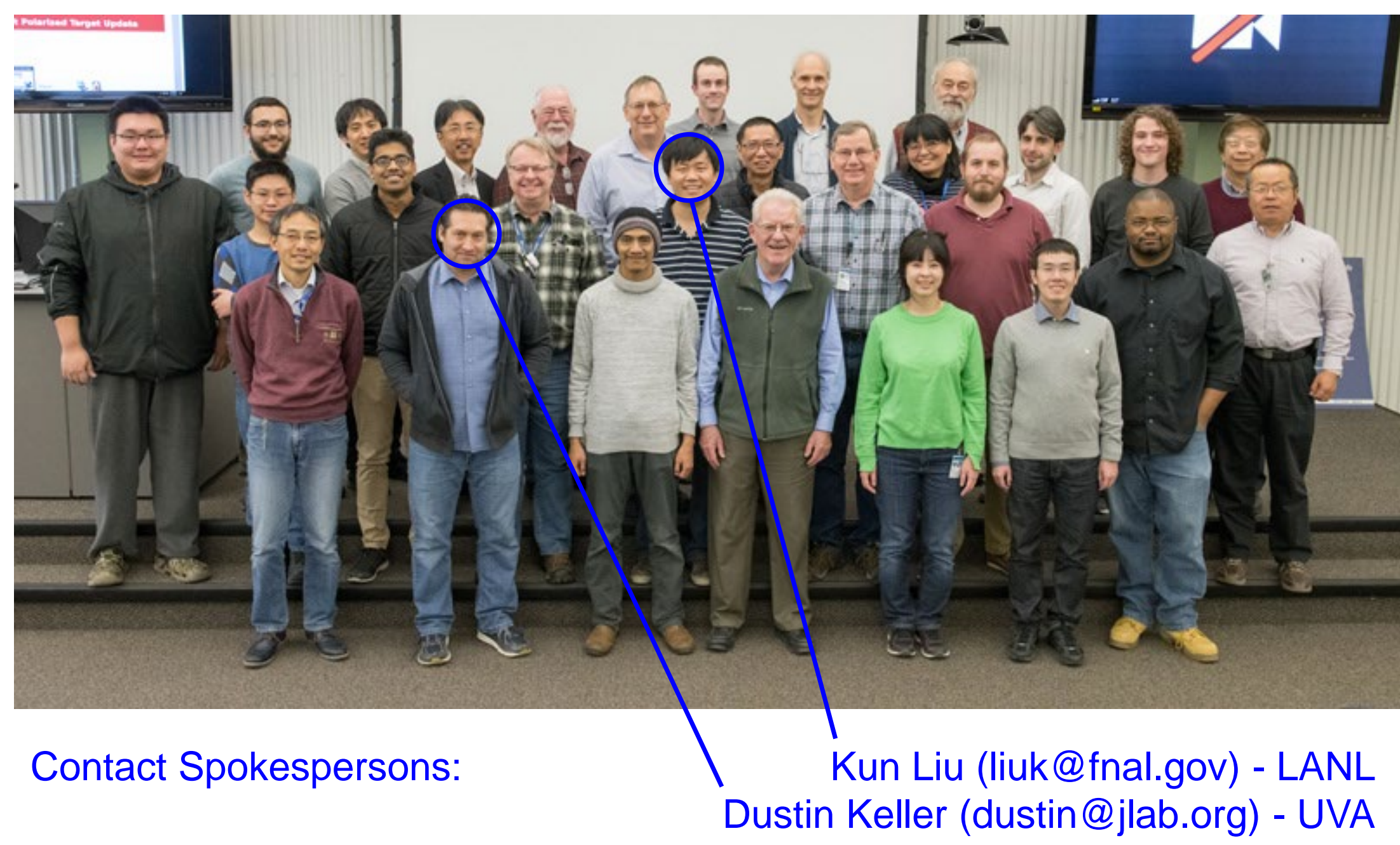

Learn more about SpinQuest/E1039:

https://spinquest.fnal.govl 


\section{Let's Polarize the Beam at Fermilab (E-1027)}

The Plan:

- Use SpinQuest Spectrometer

- Add polarized beam

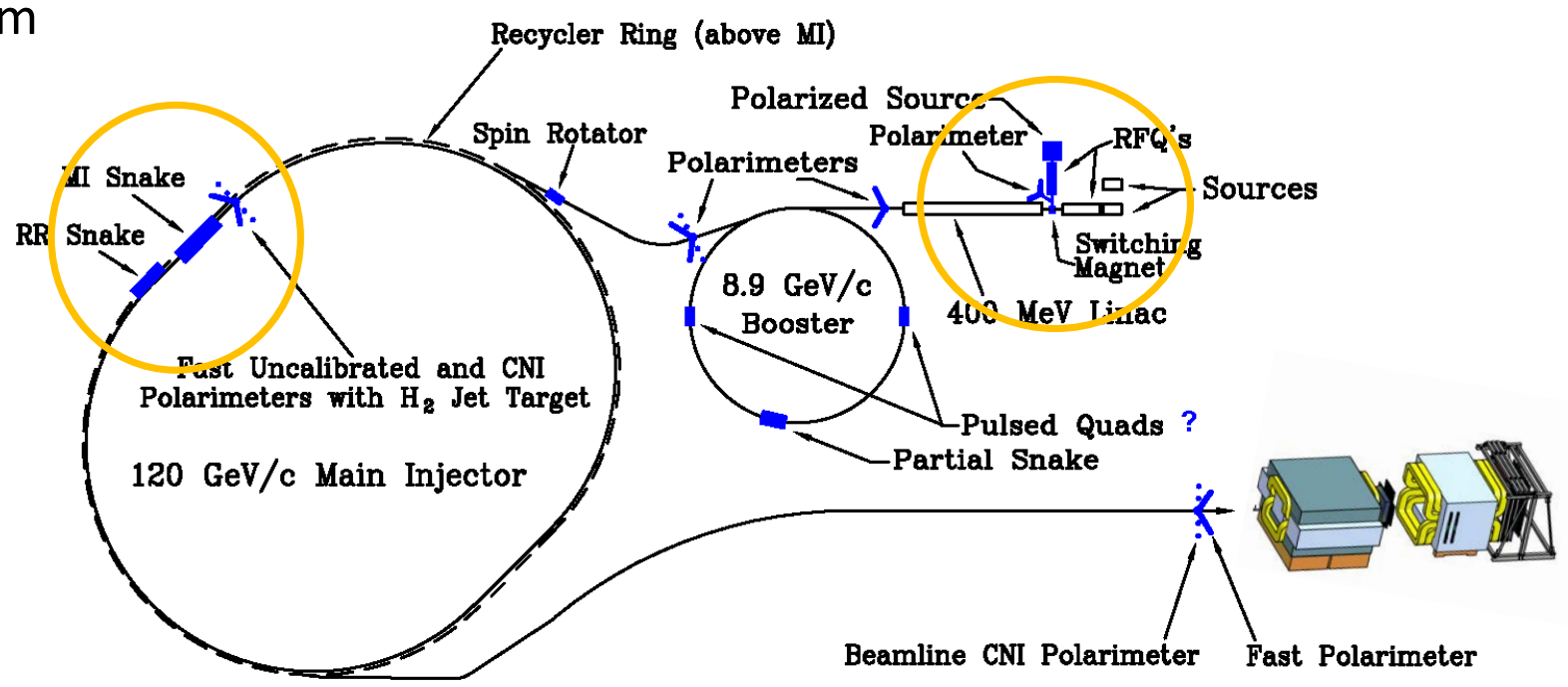

- Fermilab (best place for polarized DY):

$\longrightarrow$ very high luminosity, large $x$-coverage (primary beam, fixed target)

- Measure sign-change in Sivers Function:

$\longrightarrow$ sign, size and shape of Sivers function

$\longrightarrow$ and TMD evolution

$$
\left.f_{1 T}^{\perp}\right|_{\text {SIDIS }}=-\left.f_{1 T}^{\perp}\right|_{D Y}
$$

- Access to both valence and sea quarks

- Complementary to future EIC TMD Physics 


\section{Expected Precision from E-1027 at Fermilab}

- Probe Valence Quark Sivers Asymmetry with a polarized proton beam at SeaQuest

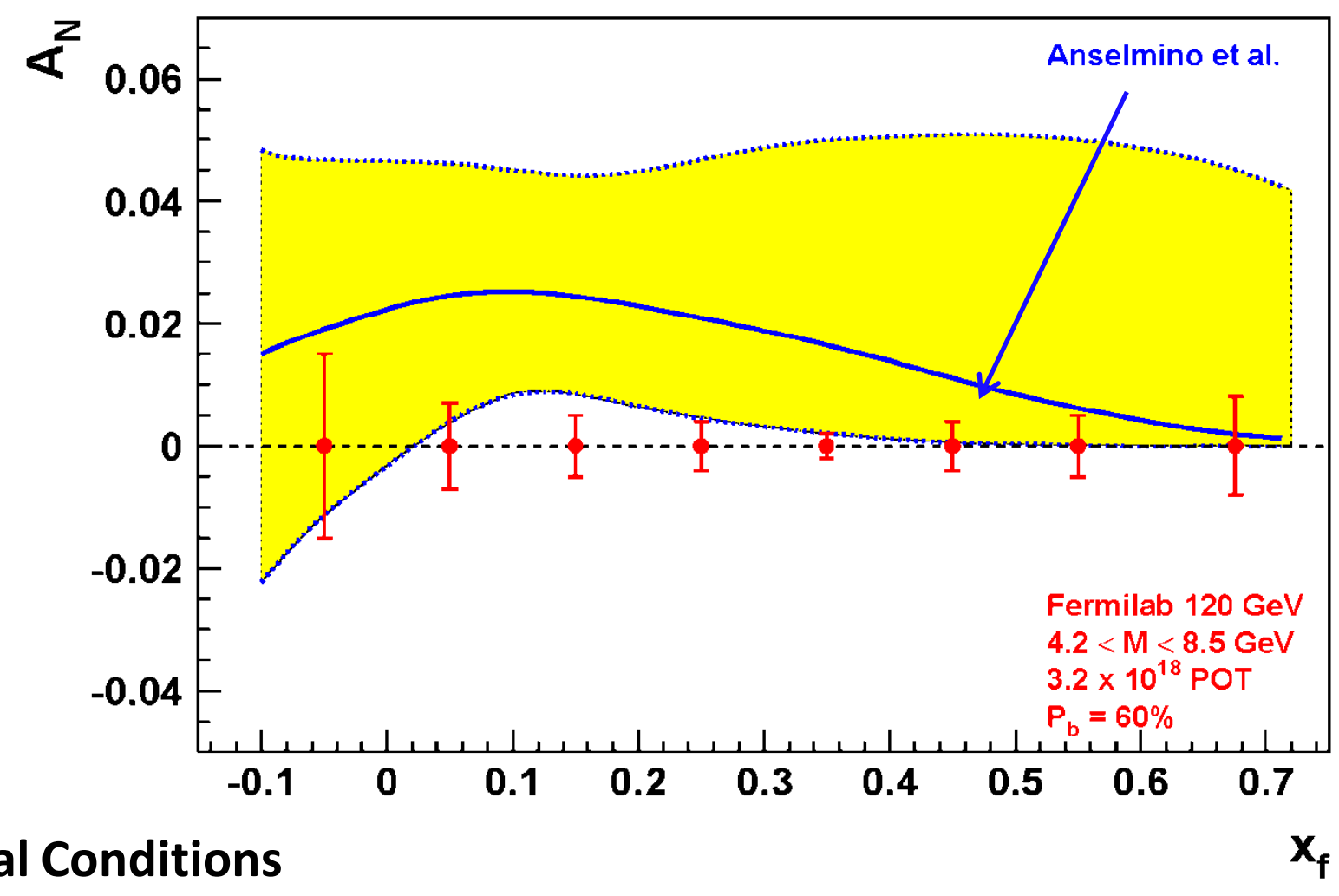

1.3 Mio DY events with no dilution

- Experimental Conditions

- same as SeaQuest

- luminosity: $L_{a v}=2 \times 10^{35}$ (10\% of available beam time: $\left.I_{a v}=15 \mathrm{nA}\right)$

- $\quad 3.2 \times 10^{18}$ total protons for $5 \times 10^{5} \mathrm{~min}$ : ( $=2 \mathrm{yrs}$ at $50 \%$ efficiency) with $\mathrm{P}_{\mathrm{b}}=60 \%$

Can measure not only sign, but also the size \& probably shape of the Sivers function! as well as TMD evolution! 


\section{Search for Dark Photons at SeaQuest}

- Classic Beam Dump Experiment

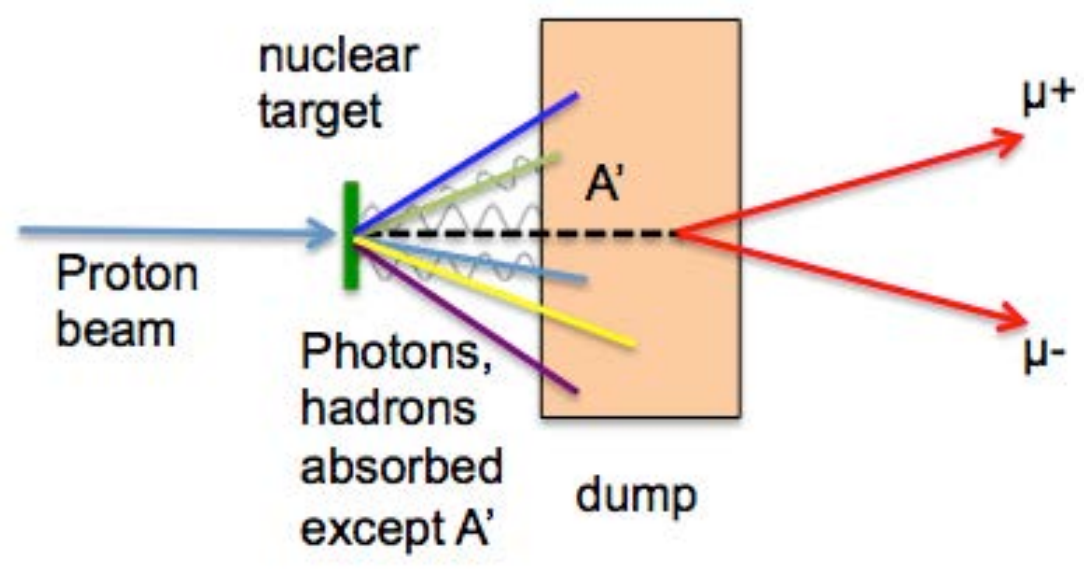

- Minimal impact on Drell-Yan program

$\rightarrow$ run parasitically during E906 \& E1039

$l_{o} \approx \frac{0.8 \mathrm{~cm}}{N_{e f f}}\left(\frac{E_{o}}{10 G e V}\right)\left(\frac{10^{-4}}{\varepsilon}\right)^{2}\left(\frac{100 \mathrm{MeV}}{m_{A}}\right)^{2}$

J. D. Bjorken et al, PRD 80 (2009) 075018

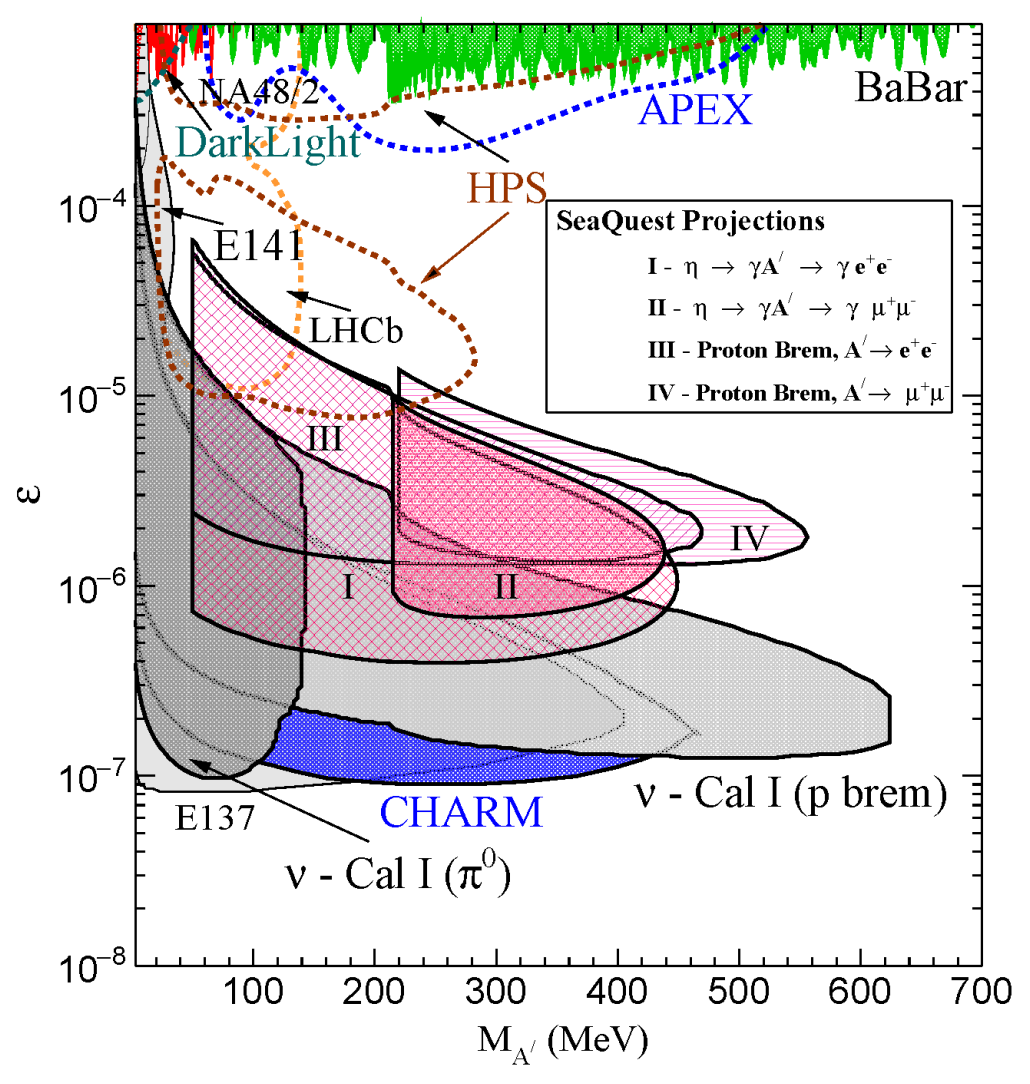

SeaQuest experimental parameters:

$\rightarrow \mathrm{E}_{0}=5-110 \mathrm{GeV}$ for Proton Bremsstrahlung

$\rightarrow \mathrm{N}_{\text {eff }}=2$

$\rightarrow \mathrm{I}_{0}=0.17 \mathrm{~m}-5.95 \mathrm{~m}$ 


\section{Polarized Proton Beams \\ and Searches for Dark Forces}

Searches for a dark photon also limit other possibilities

Parity violation studies could prove key

$$
\mathcal{L}_{\text {darkZ }}=-\left(\varepsilon e J_{\mathrm{em}}^{\mu}+\varepsilon_{Z} \frac{g}{2 \cos \theta_{W}} J_{\mathrm{NC}}^{\mu}\right) Z_{d \mu}
$$

[Davoudiasl, Lee, Marciano, 2OI4]

If the $A^{\prime}$ is a dark $Z$, then ...

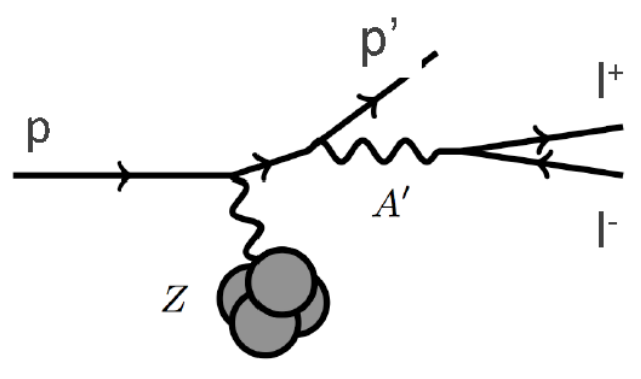

The dilepton yield can change with proton polarization: the asymmetry can be $\mathrm{O}(\mathrm{I})$ ! 


\section{Fermilab - Summary and Outlook}

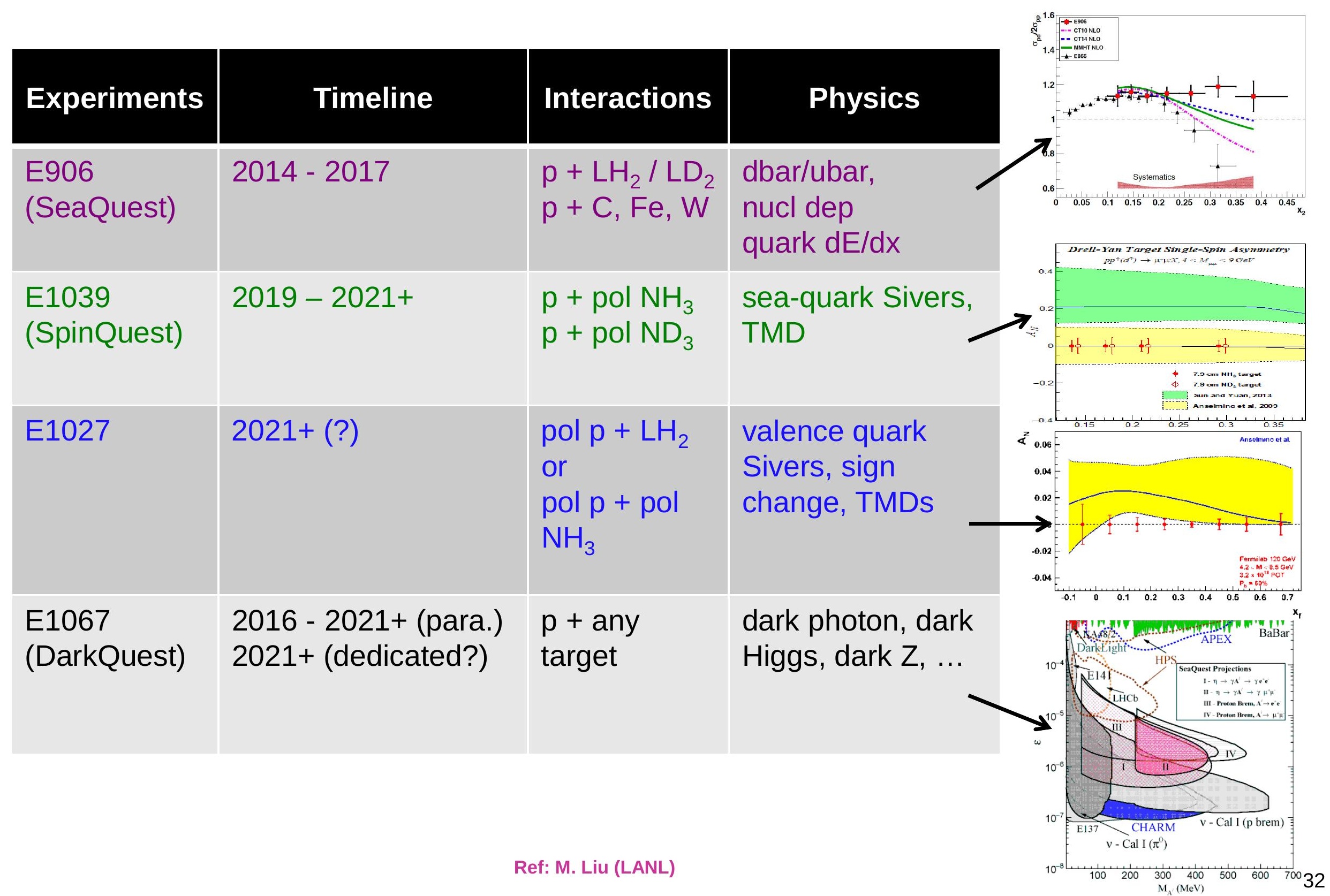




\section{Conclusions}

- There is an exciting Drell-Yan program with polarized/unpolarized beams and targets underway

$\longrightarrow$ although experimentally more challenging, it has some clear advantages over SIDIS

- Different labs offer complementary probes and processes to study hadronic landscape

$\longrightarrow$ focus on strength of each lab to (minimize cost and) optimize physics output

- Future opportunities look very promising

$\longrightarrow$ support from hadronic community (was and remains) vital to move forward

$\longrightarrow$ opportunities to join the Fermilab program

- We have seen first results from COMPASS and STAR on the sign-change

$\longrightarrow$ statistics still poor

- Now entering an era where we will have first measurement of a sea quark Sivers function (answer some of the questions):

$\longrightarrow$ How much do the quarks and gluons contribute to the nucleon spin?

$\longrightarrow$ In particular, what is the role of the sea quarks?

$\longrightarrow$ Is there significant orbital angular momentum?

$\longrightarrow$ Does TMD formalism work? Does Sivers function change sign (but keep shape and size)? 
Thank You 


\section{SpinQuest/E1039 Collaboration}

- Relatively small collaboration

$\rightarrow 36$ full members, 76 affiliate members

$\longrightarrow 14$ institutions and Fermilab
Abilene Christian University
Tokyo Institute of Technology
Argonne National Laboratory University of Colorado, Boulder
KEK
University of Illinois, Urbana-Champaign
Los Alamos National Laboratory University of Michigan
Mississippi State University
University of New Hampshire
New Mexico State University
University of Virginia
RIKEN
Yamagata University

- US collaborators supported by NSF and DOE Medium Energy

- New collaborators actively sought

$\rightarrow$ contribute and lead major detector and physics efforts

$\longrightarrow$ contact co-spokespersons 